\title{
诺蒎酮基噻唑腙类化合物的合成及对 $\alpha$-淀粉酶的抑制活性
}

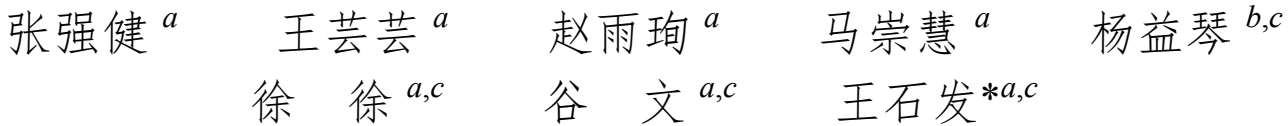 \\ ( ${ }^{a}$ 南京林业大学化学工程学院 南京 210037) \\ ( $b$ 南京林业大学轻工与食品学院 南京 210037) \\ ( ${ }^{c}$ 南京林业大学林业资源高效加工利用协同创新中心 南京 210037)
}

\begin{abstract}
摘要 以天然 $\beta$-蒎烯衍生物诺菠酮为原料, 经缩合和环化等反应, 合成了 24 个诺蒎酮基噻夾腙类化合物, 采用 ${ }^{1} \mathrm{H}$ NMR, ${ }^{13} \mathrm{C}$ NMR, HRMS 等方法对其结构进行表征, 研究了噻夾腙类化合物对 $\alpha$-淀粉酶的抑制活性. 结果表明, 与阳性 对照阿卡波糖相比，有 6 种化合物对 $\alpha$-淀粉酶表现出优良的的抑制活性，其中 4-(2-(2-(6,6-二甲基-3-(4-甲基苄亚基)双 环 [3.1.1]庚烷-2-亚基)肼基)噻唑-4-基)苯酚(SZ14)的 $\mathrm{IC}_{50}$ 值可达到 $4.11 \mu \mathrm{mol} / \mathrm{L}$. 从化合物的结构与活性关系看, $\mathrm{R}^{2}$ 的结 构对活性具有显著的影响. 抑制动力学结果表明, 这 6 种化合物是针对 $\alpha$-淀粉酶的非竞争性抑制剂. 采用分子对接方法 评价了噻唑腙类化合物与 $\alpha$-淀粉酶的结合亲和力, 并分析探索了化合物 SZ14 与 $\alpha$-淀粉酶的结合方式.
\end{abstract}

关键词＼cjkstart诺蒎酮；噻唑腙； $\alpha$-淀粉酶抑制剂; 分子对接

\section{Synthesis and $\alpha$-Amylase Inhibitory Potential and Molecular Docking Study of Nopinone-Based Thiazolylhydrazone Derivatives}

\author{
Zhang, Qiangjian ${ }^{a}$ \\ Wang, Yunyun ${ }^{a}$ \\ Zhao, Yuxun ${ }^{a}$ \\ Ma, Chonghui ${ }^{a}$ \\ Yang, Yiqing ${ }^{b, c}$ \\ $\mathrm{Xu}, \mathrm{Xu}^{a, c}$ \\ $\mathrm{Gu}, \mathrm{Wen}^{a, c}$ \\ Wang, Shifa ${ }^{*, a, c}$ \\ $\left({ }^{a}\right.$ College of Chemical Engineering, Nanjing Forestry University, Nanjing 210037) \\ $\left({ }^{b}\right.$ College of Light Industry and Food, Nanjing Forestry University, Nanjing 210037) \\ $\left({ }^{c}\right.$ Co-innovation Center of Efficient Processing and Utilization of Forest Resources, \\ Nanjing Forestry University, Nanjing 210037)
}

\begin{abstract}
A series of nopinone-based thiazolyhydrazone derivatives were synthesized by using nopinone derivated from natural $\beta$-pinene as the starting material in three steps, including aldol reaction with aromatic aldehydes, condensation with aminothiourea, and cyclization with bromoacetophenone. The structures of synthesized compounds were characterized by ${ }^{1} \mathrm{H}$ NMR, ${ }^{13} \mathrm{C}$ NMR and HRMS. $\alpha$-Amylase inhibitory activities of these compounds were also investigated. The results showed that 6 compounds among them had good inhibitory activities compared with the positive control acarbose. Especially, 4-(2-(2-(6,6-dimethyl-3-(4-methylbenzylidene))bicyclo[3.1.1]heptane-2-ylidene)indolyl-4-thiazol-4-yl)phenol (SZ14) exhibited remarkable $\alpha$-amylase inhibitory activity with $\mathrm{IC}_{50}$ value of $4.11 \mu \mathrm{mol} / \mathrm{L}$. From the structure-activity relationship, the structure of $\mathrm{R}^{2}$ gave great influence on the activities of thiazolyhydrazone derivatives. The kinetic inhibition study revealed that those 6 compounds were the noncompetitive inhibitor against $\alpha$-amylase. It was used for molecular docking study to find out binding affinities for thiazolylhydrazone derivatives, and the binding mode of compound SZ14 with $\alpha$-amylase was primarily investigated with molecular docking method.
\end{abstract}

Keywords nopinone; thiazolylhydrazone; $\alpha$-amylase inhibitor; molecular docking

糖尿病是一种以高血糖为特征的代谢性疾病, 糖尿 病还会增加患者患上其他疾病的风险 ${ }^{[1]}$. 根据世界卫生
组织(WHO)的统计, 全世界约有 4.22 亿人患有糖尿病, 其中 90\%患有 II 型糖尿病. 世界卫生组织预测, 2016 至

* Corresponding author. E-mail: wangshifa65@163.com

Received December 19, 2018; revised January 14, 2019; published online February 19, 2019.

Project supported by the National Natural Science Foundation of China (No. 31470592).

国家自然科学基金(No. 31470592)资助项目. 
2036 年糖尿病导致的死亡率将增加一倍以上 ${ }^{[2]}$. II 型糖 尿病是内分泌系统中的代谢紊乱, 患者机体对胰岛素不 敏感或者机体的胰岛素不足 ${ }^{[3 \sim 5]}$, 容易导致各种疾病, 如心血管疾病、中风、高血压、失明和肾衰竭 ${ }^{[6,7]}$. 在 II 型糖尿病患者中, 餐后血糖控制不住地上升, 会导致高 血糖以及体内代谢紊乱. 高血糖的治疗主要集中在刺激 胰 $\beta$ 细胞分泌胇岛素、抑制胰岛素降解过程、修复或再 生胰岛 $\beta$ 细胞、抑制淀粉水解酶如 $\alpha$-淀粉酶和 $\alpha$-葡萄糖 苷酶 ${ }^{[8]}$. 在目前的多种抗糖尿病的治疗方法中, $\alpha$-淀粉 酶和 $\alpha$-葡萄糖苷酶的抑制是最常用的方法 ${ }^{[9,10]}$. 目前, 常用的口服抗糖尿病药物均为 $\alpha$-淀粉酶抑制剂, 例如阿 卡波糖、米格列醇和伏格列波糖等 ${ }^{[11]}$. 这些药物可以通 过抑制 $\alpha$-淀粉酶的活性, 有效减少 II 型糖尿病患者的血 糖水平. 然而, 长期服用这类药物会导致腹部不适、腹 泻以及胃胀气等 ${ }^{[12]}$. 因此, 开发一种疗效更好且对糖尿 病治疗没有副作用的安全药物依旧是亟待解决的问题.

近年来, 关于 $\alpha$-淀粉酶抑制剂的研究越来越多, 对 天然产物的改性更是研究热点. Martinez-Gonzalez 等 ${ }^{[13]}$ 研究了不同黄酮类化合物对淀粉酶的抑制效果, 结果表 明, 橙皮素和槲皮素是竞争型抑制剂, 而木犀草素是非 竞争型抑制剂, 且具有最好的抑制效果, 但木犀草素抑 制率仍旧略差于阳性对照阿卡波糖. Taha 等 ${ }^{[14]}$ 对吲哚类 化合物进行了改性, 表明植物基生物碱经过化学改性得 到的吲哚氨基硫脲具有较好的淀粉酶抑制活性. 其中 2-(5-氯-1 $H$-吲哚-2-羰基)- $N$-(4-硝基苯基)肼-1-硫代甲酰 胺具有与阿卡波糖相当的淀粉酶抑制效果. 另外还对天 然提取物中的多酚、多糖及人工合成的吡唑啉酮类化合 物进行了 $\alpha$-淀粉酶的抑制活性研究 ${ }^{[15 ~ 17]}$, 结果表明, 此 类化合物对 $\alpha$-淀粉酶的抑制活性效果一般.
噻唑类化合物是常见的具有药学活性物质，例如磺 胺噻唑、博来霉素、噻唑弗林等均为噻唑环框架的药物. 噻唑类化合物在过敏症 ${ }^{[18]}$ 、高血压 ${ }^{[19]}$ 、炎症 ${ }^{[20] 、}$ 精神分 裂症 ${ }^{[21]}$ 、细菌感染 ${ }^{[22]}$ 、失眠症 ${ }^{[23]}$ 、人类免疫缺陷病毒 (HIV)感染 ${ }^{[24]}$ 以及血栓 ${ }^{[25]}$ 等疾病治疗方面发挥了重要的 作用. 在设计抗糖尿病药物方面, 以噻唑环作为活性框

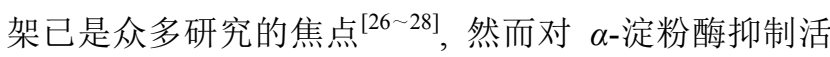
性仍有待提高. 为寻找具有高效抑制 $\alpha$-淀粉酶活性的化 合物，结合本课题组 ${ }^{[29 ~ 33]}$ 前期的工作基础，本工作以天 然 $\beta$-蒎烯衍生物诺蒎酮为先导化合物, 经缩合、环化等 反应，设计合成了 24 个新型的诺蒎酮基噻唑腙类化合 物, 采用 ${ }^{1} \mathrm{H}$ NMR, ${ }^{13} \mathrm{C}$ NMR, HRMS 等方法对其结构进 行表征, 研究了噻唑腙类化合物对 $\alpha$-淀粉酶的抑制活 性. 结果表明, 与 $\mathrm{IC}_{50}$ 值为 $10.16 \mu \mathrm{mol} / \mathrm{L}$ 的阳性对照阿 卡波糖相比，化合物 SZ13 SZ18 对 $\alpha$-淀粉酶表现出优 良的抑制活性，其中化合物 SZ14 的 $\mathrm{IC}_{50}$ 值可达到 4.11 $\mu \mathrm{mol} / \mathrm{L}$. 采用分子对接方法初步探索了化合物 SZ14 与 $\alpha$-淀粉酶的结合方式. 目标化合物的合成路线见 Scheme 1 .

\section{1 结果与讨论}

\section{1 合成与表征}

诺蒎酮基噻唑腙类衍生物 $\mathbf{S Z 1} \sim \mathbf{S Z 2 4}$ 的合成包括 三步：羟醛缩合、加成缩合和 Hantzsch 环化反应. 化合 物 1 与对取代苯甲醛发生羟醛缩合，制备中间体 2; 以 盐酸为催化剂，中间体 $\mathbf{2}$ 和氨基硫脲缩合制得中间体 $\mathbf{3}$; 中间体 3 与对取代-2-溴苯乙酮进行环化制得噻唑腙类 化合物 SZ1 SZ24. 采用 ${ }^{1} \mathrm{H}$ NMR, ${ }^{13} \mathrm{C}$ NMR 和 HRMS
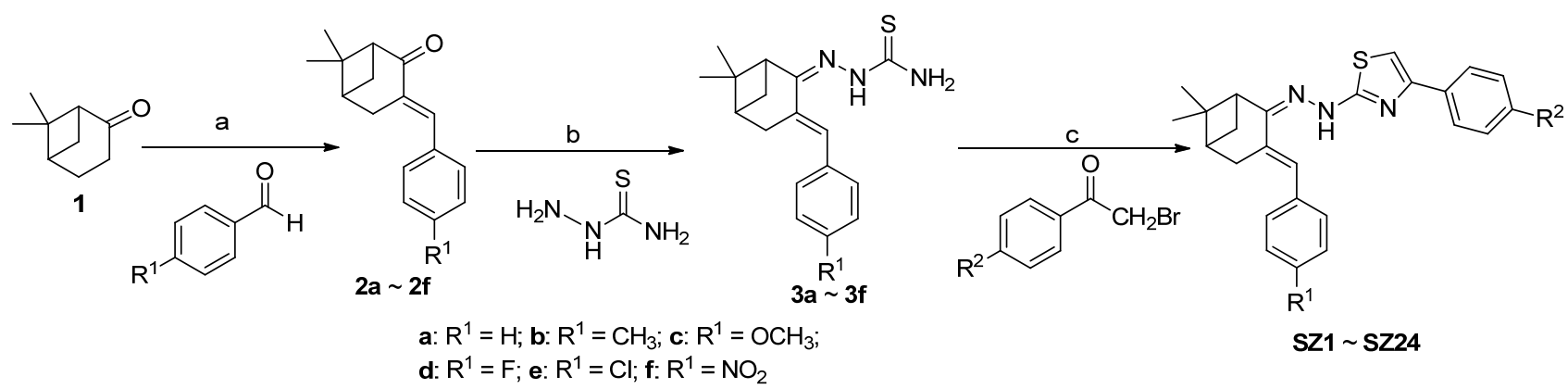

d: $\mathrm{R}^{1}=\mathrm{F} ; \mathbf{e}: \mathrm{R}^{1}=\mathrm{Cl} ; \mathbf{f}: \mathrm{R}^{1}=\mathrm{NO}_{2}$

SZ1 SZ24

SZ1: $R^{1}=H, R^{2}=H ;$ SZ2: $R^{1}=C_{3}, R^{2}=H ;$ SZ3: $R^{1}=O_{3}, R^{2}=H ; S Z 4: R^{1}=F, R^{2}=H ;$ SZ5: $R^{1}=C l, R^{2}=H$;

SZ6: $R^{1}=\mathrm{NO}_{2}, \mathrm{R}^{2}=\mathrm{H} ; \mathrm{SZ7}: \mathrm{R}^{1}=\mathrm{H}, \mathrm{R}^{2}=\mathrm{OCH}_{3}$; SZ8: $\mathrm{R}^{1}=\mathrm{CH}_{3}, \mathrm{R}^{2}=\mathrm{OCH}_{3} ; \mathrm{SZ9}: \mathrm{R}^{1}=\mathrm{OCH}_{3}, \mathrm{R}^{2}=\mathrm{OCH}_{3}$;

SZ10: $R^{1}=\mathrm{F}, \mathrm{R}^{2}=\mathrm{OCH}_{3} ;$ SZ11: $\mathrm{R}^{1}=\mathrm{Cl}, \mathrm{R}^{2}=\mathrm{OCH}_{3} ; \mathbf{S Z 1 2}: \mathrm{R}^{1}=\mathrm{NO}_{2}, \mathrm{R}_{2}=\mathrm{OCH}_{3} ; \mathbf{S Z 1 3}: \mathrm{R}^{1}=\mathrm{H}, \mathrm{R}^{2}=\mathrm{OH}$;

SZ14: $R^{1}=\mathrm{CH}_{3}, \mathrm{R}^{2}=\mathrm{OH}$; SZ15: $\mathrm{R}^{1}=\mathrm{OCH}_{3}, \mathrm{R}^{2}=\mathrm{OH}$; SZ16: $\mathrm{R}^{1}=\mathrm{F}, \mathrm{R}^{2}=\mathrm{OH}$; SZ17: $\mathrm{R}^{1}=\mathrm{Cl}, \mathrm{R}^{2}=\mathrm{OH}$;

SZ18: $R^{1}=\mathrm{NO}_{2}, R^{2}=\mathrm{OH} ; \mathbf{S Z 1 9}: \mathrm{R}^{1}=\mathrm{H}, \mathrm{R}^{2}=\mathrm{F} ; \mathbf{S Z 2 O}: \mathrm{R}^{1}=\mathrm{CH}_{3}, \mathrm{R}^{2}=\mathrm{F} ; \mathbf{S Z 2 1}: \mathrm{R}^{1}=\mathrm{OCH}_{3}, \mathrm{R}^{2}=\mathrm{F}$;

SZ22: $R^{1}=F, R^{2}=F ;$ SZ23: $R^{1}=\mathrm{Cl}, R^{2}=F ;$ SZ24: $^{1}=\mathrm{NO}_{2}, R^{2}=F$

Reagents and conditions: (a) aromatic benzaldehydes, $\mathrm{C}_{2} \mathrm{H}_{5} \mathrm{ONa}$, ethanol, reflux, $8 \mathrm{~h}$; (b) $\mathrm{HCl}$, ethanol, reflux, $4 \sim 6 \mathrm{~h}$; (c) bromoacetophenone, reflux.

图式 1 诺溗酮基噻唑腙类衍生物 SZ1 SZ24 的合成

Scheme 1 Synthesis of nopinone-based thiazolylhydrazone derivatives SZ1 SZ24 
等手段对目标化合物的结构进行表征. 例如化合物 SZ22 的 ${ }^{1} \mathrm{H}$ NMR 分析结果表明, 在 $\delta 7.24 \sim 7.87$ 处观察 到对应于芳族质子的单峰和双峰, 在 $\delta 6.89$ 处发现了噻 唑环的质子峰. $\mathrm{NH}$ 的质子峰在 $\delta 12.25$ 处出现单峰. SZ22 的 ${ }^{13} \mathrm{C}$ NMR 谱显示, 芳族碳的峰在 $\delta 115.95 \sim$ 132.27 处, 在 $\delta 100.77,169.74$ 和 165.40 出现的峰证明了 噻唑环的存在.

诺蒎酮与取代苯甲醛的羟醛缩合反应中, 取代基 $\mathrm{R}^{1}$ 的类型对反应有显著影响. 当 $\mathrm{R}^{1}$ 为供电子基时的反 应速度略低于为吸电子基时的反应速度, 表现在反应时 间延长且产物的得率略低. 主要是因为供电子基的存在 导致羰基碳的电子云密度增高, 其亲电能力降低, 不利 于羟醛缩合反应的进行. 但 $\mathrm{R}^{1}$ 的结构对不饱和酮与氨 基硫嫝的缩合反应影响不明显. 中间体 $\mathbf{3}$ 与对取代-2-溴 苯乙酮的环化反应极易进行, $\mathrm{R}^{2}$ 的结构对环化反应基本 没有影响.

\section{2 生物活性测定}

诺蒎酮基噻唑腙类化合物对 $\alpha$-淀粉酶的半抑制浓 度 $\left(\mathrm{IC}_{50}\right)$ 见表 1. 由表 1 可知, 化合物 $\mathbf{S Z 1 3} \sim \mathbf{S Z 1 8}$ 对 $\alpha$ 淀粉酶表现出极强的抑制效果, 尤其是化合物 SZ14,

表 1 化合物 SZ1 SZ24 对 $\alpha$-淀粉酶的半抑制浓度 $\left[\left(\mathrm{IC}_{50} /\right.\right.$ $\left.\left(\mu \mathrm{mol} \cdot \mathrm{L}^{-1}\right)\right]^{a}$

Table 1 Inhibitory activity of compounds SZ1 $\sim$ SZ24 against $\alpha$-amylase $\left[\mathrm{IC}_{50} /\left(\mu \mathrm{mol} \cdot \mathrm{L}^{-1}\right)\right]$

\begin{tabular}{|c|c|c|c|c|}
\hline Compd. & $\mathrm{R}^{1}$ & $\mathrm{R}^{2}$ & 产率/\% & $\mathrm{IC}_{50} /\left(\mu \mathrm{mol} \cdot \mathrm{L}^{-1}\right)$ \\
\hline SZ1 & $\mathrm{H}$ & $\mathrm{H}$ & 77.6 & $208.20 \pm 0.06$ \\
\hline SZ2 & $p-\mathrm{CH}_{3}$ & $\mathrm{H}$ & 85.3 & $174.83 \pm 0.11$ \\
\hline SZ3 & $p-\mathrm{OCH}_{3}$ & $\mathrm{H}$ & 83.6 & $156.32 \pm 0.05$ \\
\hline SZ4 & $p-\mathrm{F}$ & $\mathrm{H}$ & 74.2 & $237.15 \pm 0.09$ \\
\hline SZ5 & $p-\mathrm{Cl}$ & $\mathrm{H}$ & 77.6 & $>250$ \\
\hline SZ6 & $p-\mathrm{NO}_{2}$ & $\mathrm{H}$ & 87.5 & $94.22 \pm 0.07$ \\
\hline SZ7 & $\mathrm{H}$ & $p-\mathrm{OCH}_{3}$ & 83.3 & $178.73 \pm 0.11$ \\
\hline SZ8 & $p-\mathrm{CH}_{3}$ & $p-\mathrm{OCH}_{3}$ & 82.6 & $>250$ \\
\hline SZ9 & $p-\mathrm{OCH}_{3}$ & $p-\mathrm{OCH}_{3}$ & 82.1 & $167.05 \pm 0.06$ \\
\hline SZ10 & $p-\mathrm{F}$ & $p-\mathrm{OCH}_{3}$ & 82.3 & $>250$ \\
\hline SZ11 & $p-\mathrm{Cl}$ & $p-\mathrm{OCH}_{3}$ & 83.5 & $25.78 \pm 0.05$ \\
\hline SZ12 & $p-\mathrm{NO}_{2}$ & $p-\mathrm{OCH}_{3}$ & 83.8 & $56.60 \pm 0.09$ \\
\hline SZ13 & $\mathrm{H}$ & $p-\mathrm{OH}$ & 70.2 & $4.42 \pm 0.01$ \\
\hline SZ14 & $p-\mathrm{CH}_{3}$ & $p-\mathrm{OH}$ & 73.4 & $4.11 \pm 0.01$ \\
\hline SZ15 & $p-\mathrm{OCH}_{3}$ & $p-\mathrm{OH}$ & 72.7 & $4.68 \pm 0.02$ \\
\hline SZ16 & $p-\mathrm{F}$ & $p-\mathrm{OH}$ & 81.5 & $5.38 \pm 0.02$ \\
\hline SZ17 & $p-\mathrm{Cl}$ & $p-\mathrm{OH}$ & 66.1 & $5.08 \pm 0.01$ \\
\hline SZ18 & $p-\mathrm{NO}_{2}$ & $p-\mathrm{OH}$ & 64.2 & $5.43 \pm 0.01$ \\
\hline SZ19 & $\mathrm{H}$ & $p-\mathrm{F}$ & 83.9 & $148.91 \pm 0.05$ \\
\hline SZ20 & $p-\mathrm{CH}_{3}$ & $p-\mathrm{F}$ & 79.0 & $>250$ \\
\hline SZ21 & $p-\mathrm{OCH}_{3}$ & $p-\mathrm{F}$ & 82.7 & $>250$ \\
\hline SZ22 & $p-\mathrm{F}$ & $p-\mathrm{F}$ & 71.2 & $>250$ \\
\hline SZ23 & $p-\mathrm{Cl}$ & $p-\mathrm{F}$ & 75.3 & $>250$ \\
\hline SZ24 & $p-\mathrm{NO}_{2}$ & $p-\mathrm{F}$ & 73.2 & $96.89 \pm 0.06$ \\
\hline 阿卡波糖 & & & & $10.16 \pm 0.05$ \\
\hline
\end{tabular}

其 $\mathrm{IC}_{50}$ 为 $4.11 \mu \mathrm{mol} / \mathrm{L}$, 抑制活性是阳性对照的 2.47 倍 (阳性对照阿卡波糖的 $\mathrm{IC}_{50}$ 为 $10.16 \mu \mathrm{mol} / \mathrm{L}$ ). SZ19 SZ24 对 $\alpha$-淀粉酶几乎没有抑制效果. 从化合物的结构 与活性关系看, $\mathrm{R}^{1}$ 的结构对化合物活性影响并不明显, 而 $\mathrm{R}^{2}$ 的结构则对化合物活性有显著影响. 当 $\mathrm{R}^{2}$ 为羟基 时，化合物对 $\alpha$-淀粉酶具有显著的抑制活性，其抑制活 性优于阳性对照药阿卡波糖; 而当 $\mathrm{R}^{2}$ 为氟时, 化合物对 $\alpha$-淀粉酶没有抑制活性; 当 $\mathrm{R}^{2}$ 为甲氧基或氢时，化合物 对 $\alpha$-淀粉酶的抑制活性较弱.

麦芽糖在二硝基水杨酸试剂(DNSA)染色煮沸后吸 光值的标准曲线回归方程为 $y=3.361 x+0.012, R^{2}=$ 0.986 , 吸光度在 $540 \mathrm{~nm}$ 处, 麦芽糖浓度 $0.1 \sim 1$ $\mathrm{mmol} / \mathrm{mL}$ 范围内, 线性关系良好(图 1).

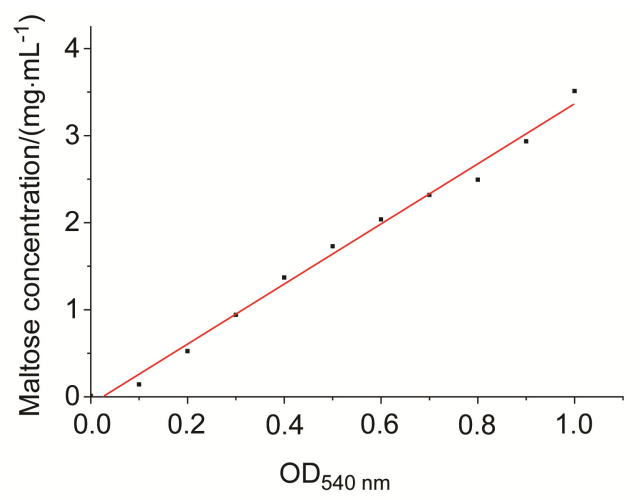

图 1 麦芽糖标准曲线图

Figure 1 Maltose standard plot

采用动力学方法对化合物 SZ13 SZ18 抑制 $\alpha$-淀粉 酶的抑制类型进行了分析. 以 $1 / v$ 为纵坐标, $1 /[\mathrm{S}]$ 为横坐 标，作 Lineweaver-Burk 曲线图，如图 2 所示. 不同底物 浓度得到的直线交于横坐标轴属于非竞争性抑制，相交 但不交于横坐标轴属于竞争性抑制，平行则为反竞争性 抑制. 由图 2 可以看出, SZ13 SZ18 均是针对 $\alpha$-淀粉酶 的非竞争性抑制，这个结果与常用阳性对照阿卡波糖的 抑制类型一致 ${ }^{[35]}$.

\section{3 作用机制研究}

为了探究噻唑腙类化合物与 $\alpha$-淀粉酶的结合模式, 分别研究了化合物 $\mathbf{S Z 1 3}$ SZ18 与胰淀粉酶(PDB ID: $3 \mathrm{bc} 9$ )的分子对接 ${ }^{[36]}$. 结果表明, 化合物 SZ13 SZ18 与 3 bc9 具有高结合亲和力, 与活性测定结果相一致. 化合 物 SZ13 SZ18 和阳性对照阿卡波糖的结合亲和力数据 如表 2 所示, 化合物 SZ14 和阿卡波糖与 $\alpha$-淀粉酶的相 互作用模式如图 3 所示. 由表 2 可知, 化合物 SZ13 SZ18 与 $3 b c 9$ 对接打分均在 7.5 以上, 而阿卡波糖与 $3 \mathrm{bc9}$ 的打分为 7.0, 说明化合物 SZ13 SZ18 表现出对 $\alpha$-淀粉酶的高亲和力. 由图 3A 所示的 SZ14 与 $\alpha$-淀粉 

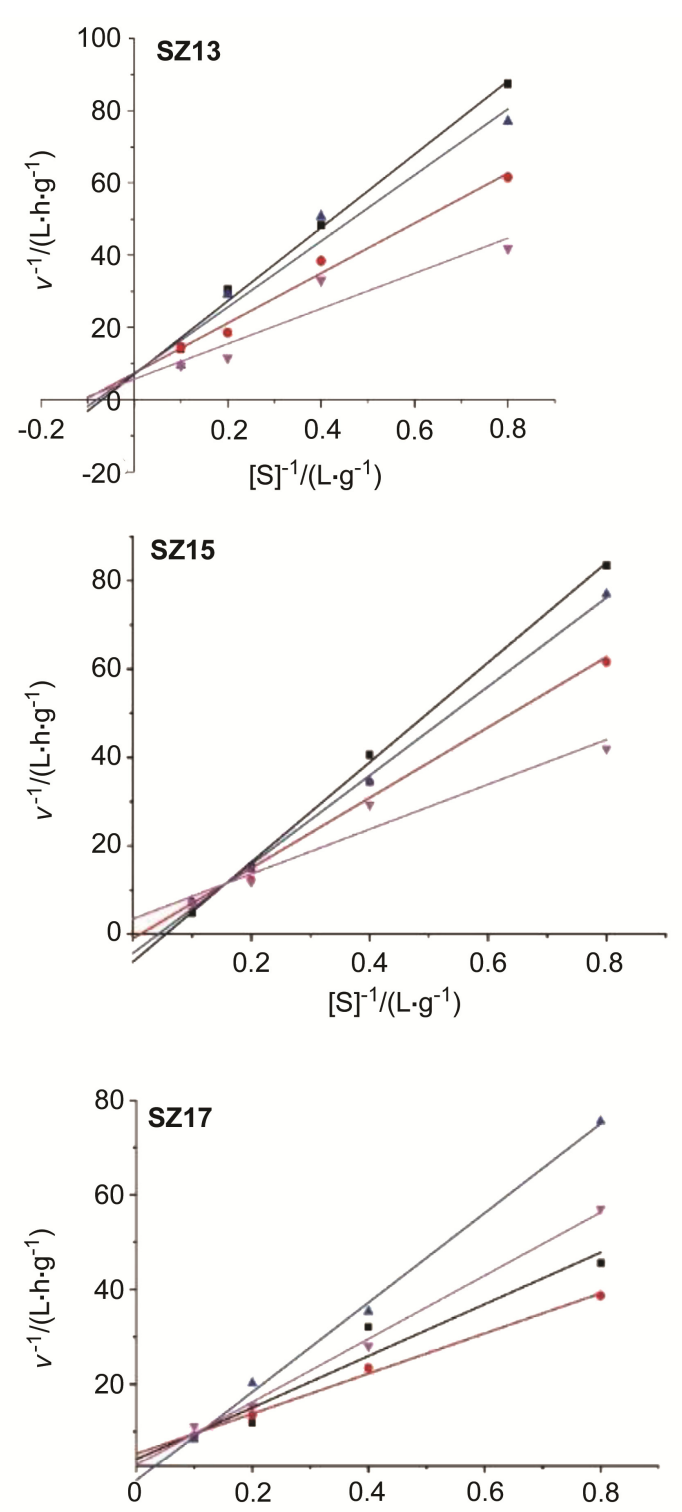

$[\mathrm{S}]^{-1} /\left(\mathrm{L} \cdot \mathrm{g}^{-1}\right)$

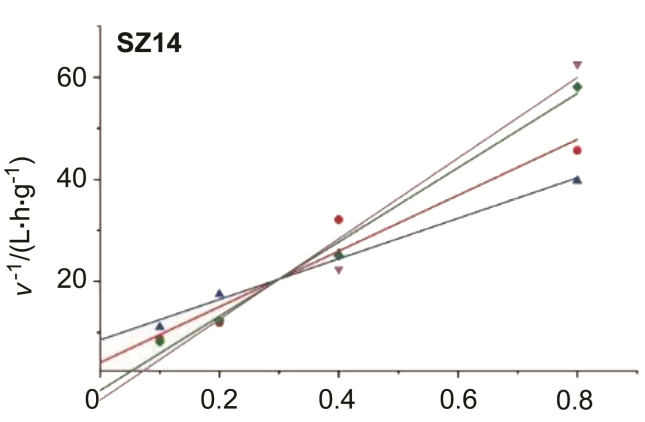

$[\mathrm{S}]^{-1 /\left(L \cdot \mathrm{g}^{-1}\right)}$

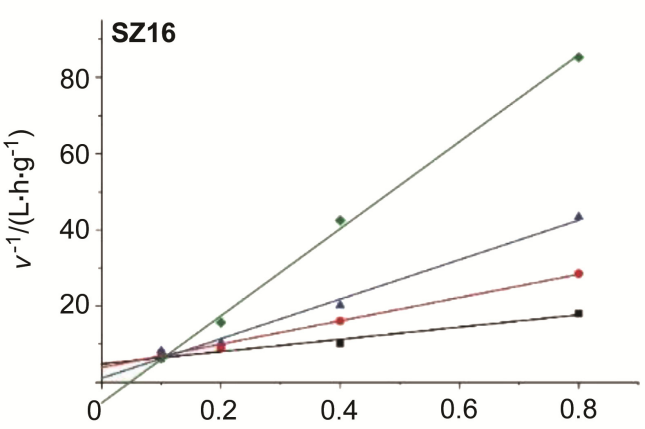

$[\mathrm{S}]^{-1} /\left(\mathrm{L} \cdot \mathrm{g}^{-1}\right)$

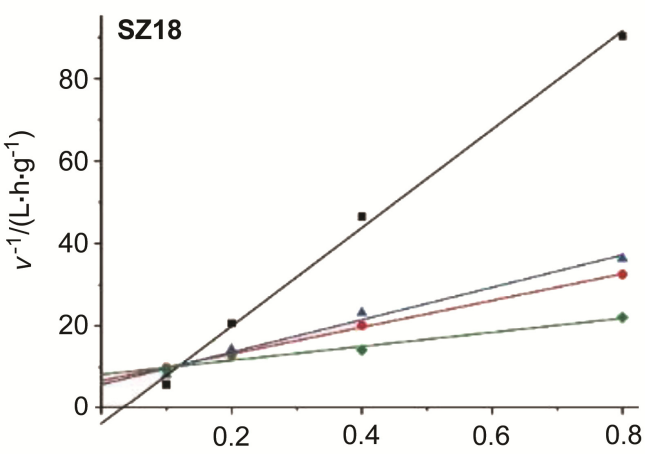

$[S]^{-1} /\left(L \cdot g^{-1}\right)$

图 2 化合物 SZ13 SZ18 对 $\alpha$-淀粉酶的 Lineweaver-Burk 曲线图

Figure 2 Lineweaver-Burk plot of compounds $\mathbf{S Z 1 3} \sim \mathbf{S Z 1 8}$ against $\alpha$-amylase

The square, round, triangle, inverted triangle and diamond symbols represent compounds $\mathbf{S Z 1 3} \sim \mathbf{S Z 1 8}$ concentrations of $40,20,10 \mathrm{and} 2.5 \mu \mathrm{g} / \mathrm{mL}$, respectively

酶的相互作用模式可知, 噻唑环上的硫原子分别与 SER-458 及 TRP488 形成氢键相互作用; 苯环与 $\alpha$-淀粉 酶 TYR-460 形成 $\pi-\pi$ 堆积, 噻唑环相接的肼基与 ASP-449, MET-490 以及 TRP488 产生强静电相互作用, 使得 SZ14 对 $\alpha$-淀粉酶具有高结合亲和力. 图 3B 中展示 了阿卡波糖的末端羟基与 ASP-449，TYR-460，ILE-459, LYS-463, TYR-464 和 ASP-589 形成氢键, 成键类型单 一，导致阿卡波糖的结合亲和力弱于 SZ14.

\section{2 结论}

以诺蒎酮为原料合成了 24 个诺蒎酮基噻唑腙类化
表 2 诺蒎酮基塞唑腙类化合物对 $3 b c 9$ 的结合亲和力 Table 2 Selected nopinone-based thiazolyhydrazone derivatives based on binding affinity to $3 \mathrm{bc} 9$

\begin{tabular}{cc}
\hline Compd. & Affinity $/\left(\mathrm{kJ} \cdot \mathrm{mol}^{-1}\right)$ \\
\hline SZ13 & -32.2 \\
SZ14 & -32.6 \\
SZ15 & -31.4 \\
SZ16 & -33.9 \\
SZ17 & -31.8 \\
SZ18 & -31.4 \\
卡波糖 & -29.3 \\
\hline
\end{tabular}



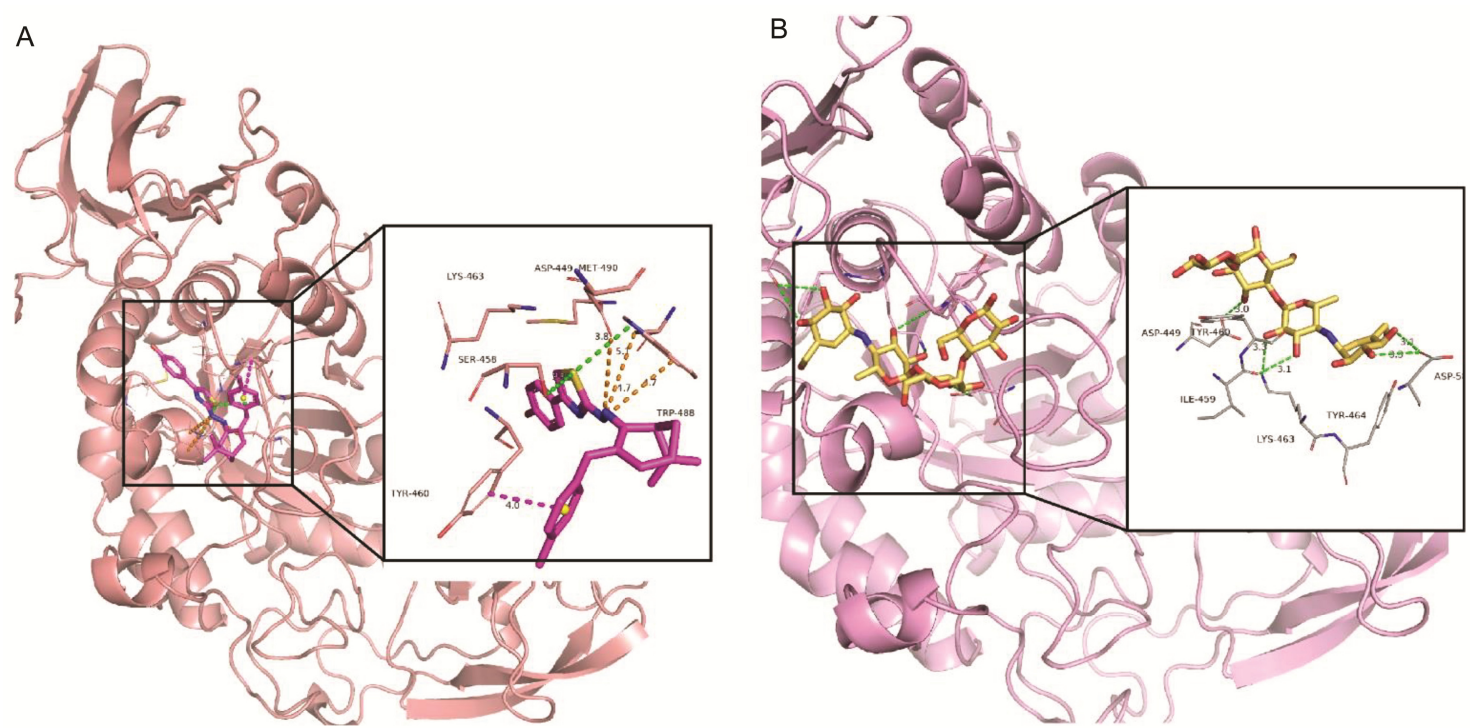

图 3 (A)化合物 SZ14 和(B)阿卡波糖与 $3 b c 9$ 的分子结合方式

Figure 3 Binding mode of compounds SZ14 (A) and acarbose (B) with 3bc9

合物，并且采用 ${ }^{1} \mathrm{H} N M R,{ }^{13} \mathrm{C} \mathrm{NMR}$ 和 HRMS 对化合物 进行了结构确认. $\alpha$-淀粉酶抑制实验表明, SZ13 SZ18 对 $\alpha$-淀粉酶抑制效果优于阳性对照阿卡波糖 $\left(\mathrm{IC}_{50}\right.$ 值为 $10.16 \mu \mathrm{mol} / \mathrm{L})$. 特别是 $\mathbf{S Z 1 4}$, 抑制效果为阿卡波糖的 2.47 倍. 探索了上述 6 种化合物对 $\alpha$-淀粉酶的抑制类型, 发现均为非竞争性抑制, 与阳性对照相同. 通过分子对 接实验发现, SZ13 SZ18 和阿卡波糖具有相似的活性 位点，且更易与 $\alpha$-淀粉酶结合.

\section{3 实验部分}

\section{1 仪器与试剂}

DF-101S 型恒温磁力搅拌器, 南京科尔公司; 美国 Agilent 5975c 质谱仪; 德国 Bruker AV 500 核磁共振仪 (TMS 为内标, $\mathrm{CDCl}_{3}$ 或 $\mathrm{C}_{5} \mathrm{D}_{5} \mathrm{~N}$ 为溶剂); 美国 OptiMelt 全自动熔点仪; UV-2450 紫外分光光度计 (日本 Shimadzu); 美国 Agilent 6540 高分辨质谱仪.

$\beta$-蒎烯(江西宏达天然香料有限公司, 质量分数为 $91.5 \%$; 诺蒎酮由 $\beta$-蒎烯合成 ${ }^{[37]}$; 柱色谱的硅胶 $(200 \sim$ 300 目)购自青岛海洋化学工厂; $\alpha$-淀粉酶取自猪胰腺; 其他试剂均为市售分析纯.

\section{2 化合物 2 的制备}

根据文献[34]报道, 取乙醇钠 $(14.5 \mathrm{mmol})$ 和相应的 取代苯甲醛(1.1 equiv., $15.9 \mathrm{~mol})$ 加入到诺蒎酮 1 (14.5 $\mathrm{mmol}$ )的乙醇溶液中, 并将所得混合物搅拌回流 $7.5 \mathrm{~h}$. 冷却后, 减压蒸发除去溶剂. 通过硅胶(200 300目) 柱 色谱分离粗产物[淋洗剂: 石油醚/乙酸乙酯 $(V: V=3$ : 1)]得到化合物 $\mathbf{2} \mathbf{a} \sim \mathbf{2 f}^{[29]}$.

(E)-3-茮基-6,6-二甲基二环[3.1.1]庚烷-2-酮(2a)：无
色透明晶体，产率 $78.3 \%$. m.p. $108.4 \sim 108.9{ }^{\circ} \mathrm{C} ;{ }^{1} \mathrm{H}$ NMR (400 MHz, $\left.\mathrm{CDCl}_{3}\right) \delta: 7.70(\mathrm{~s}, 1 \mathrm{H}), 7.58$ (d, $J=8.4$ $\mathrm{Hz}, 2 \mathrm{H}), 7.50 \sim 7.31(\mathrm{~m}, 3 \mathrm{H}), 2.98(\mathrm{t}, J=5.4 \mathrm{~Hz}, 2 \mathrm{H}), 2.69$ $(\mathrm{t}, J=6.0 \mathrm{~Hz}, 1 \mathrm{H}), 2.62 \sim 2.58(\mathrm{~m}, 1 \mathrm{H}), 2.38 \sim 2.33(\mathrm{~m}$, $1 \mathrm{H}), 1.56$ (d, $J=11.1 \mathrm{~Hz}, 1 \mathrm{H}), 1.38$ (s, 3H), 0.93 (s, 3H); ${ }^{13} \mathrm{C}$ NMR (100 MHz, $\left.\mathrm{CDCl}_{3}\right) \delta: 203.32,135.61,132.57$, 130.66, 128.81, 128.47, 55.79, 40.73, 39.32, 30.81, 27.35, $26.12,21.55$.

(E)-6,6-二甲基-3-(4-甲基苄亚基)二环[3.1.1]庚烷-2酮(2b): 无色透明晶体, 产率 85.7\%. m.p. 95.2 95.8 ${ }^{\circ} \mathrm{C}$ (lit. $\left.{ }^{[29]} 95 \sim 96{ }^{\circ} \mathrm{C}\right) ;{ }^{1} \mathrm{H}$ NMR $\left(400 \mathrm{MHz}, \mathrm{CDCl}_{3}\right) \delta: 7.68$ (s, $1 \mathrm{H}$ ), 7.49 (d, $J=7.7 \mathrm{~Hz}, 2 \mathrm{H}), 7.24$ (t, $J=8.0 \mathrm{~Hz}, 2 \mathrm{H})$, $2.96(\mathrm{~s}, 2 \mathrm{H}), 2.69(\mathrm{t}, J=5.6 \mathrm{~Hz}, 1 \mathrm{H}), 2.63 \sim 2.60(\mathrm{~m}, 1 \mathrm{H})$, $2.36 \sim 2.34(\mathrm{~m}, 1 \mathrm{H}), 1.50(\mathrm{~d}, J=6.0 \mathrm{~Hz}, 1 \mathrm{H}), 1.38(\mathrm{~s}, 3 \mathrm{H})$, $0.92(\mathrm{~s}, 3 \mathrm{H}) ;{ }^{13} \mathrm{C} \mathrm{NMR}\left(100 \mathrm{MHz}, \mathrm{CDCl}_{3}\right) \delta: 203.44$, $139.16,135.68,132.94,131.70,130.79$, 129.29, 55.88, 40.89, 39.46, 30.95, 27.48, 26.19, 21.58, 21.33.

(E)-3-(4-甲氧基苄亚基)-6,6-二甲基二环 [3.1.1]庚 烷-2-酮(2c): 无色透明晶体，产率 83.6\%. m.p. 82.7 $83.8{ }^{\circ} \mathrm{C}$ (lit. ${ }^{[29]} 82 \sim 83{ }^{\circ} \mathrm{C}$ ); ${ }^{1} \mathrm{H}$ NMR (400 MHz, $\mathrm{CDCl}_{3}$ ) $\delta: 7.60(\mathrm{~d}, J=8.8 \mathrm{~Hz}, 2 \mathrm{H}), 7.00(\mathrm{~d}, J=8.8 \mathrm{~Hz}, 2 \mathrm{H}), 7.52$ (s, 1H), 3.79 (s, 3H), $2.87(\mathrm{~d}, J=2.1 \mathrm{~Hz}, 2 \mathrm{H}), 2.58(\mathrm{t}, J=$ $5.2 \mathrm{~Hz}, 1 \mathrm{H}), 2.52(\mathrm{t}, J=5.6 \mathrm{~Hz}, 1 \mathrm{H}), 2.30 \sim 2.28(\mathrm{~m}, 1 \mathrm{H})$, $1.36(\mathrm{~d}, J=10.3 \mathrm{~Hz}, 1 \mathrm{H}), 1.31(\mathrm{~s}, 3 \mathrm{H}), 0.80(\mathrm{~s}, 3 \mathrm{H}) ;{ }^{13} \mathrm{C}$ NMR (100 MHz, $\left.\mathrm{CDCl}_{3}\right) \delta$ : 201.50, 159.87, 134.16, $132.43,130.19,127.71,114.15,55.15,55.13,40.10,38.87$, $30.39,26.91,25.57,21.19$.

(E)-3-(4-氟苠亚基)-6,6-二甲基二环[3.1.1]庚烷-2-酮 (2d): 无色透明晶体, 产率 $90.0 \%$. m.p. 90.8 91.5 ${ }^{\circ} \mathrm{C}$ 
(lit. $\left.{ }^{[29]} 91 \sim 92{ }^{\circ} \mathrm{C}\right) ;{ }^{1} \mathrm{H}$ NMR (400 MHz, $\mathrm{CDCl}_{3}$ ) $\delta: 7.67$ (s, $1 \mathrm{H}), 7.61 \sim 7.56(\mathrm{~m}, 2 \mathrm{H}), 7.14 \sim 7.08(\mathrm{~m}, 2 \mathrm{H}), 2.95(\mathrm{~s}$, $2 \mathrm{H}), 2.73 \sim 2.60(\mathrm{~m}, 2 \mathrm{H}), 2.41 \sim 2.35(\mathrm{~m}, 1 \mathrm{H}), 1.53 \sim 1.49$ (d, $J=10.4 \mathrm{~Hz}, 1 \mathrm{H}), 1.39$ (s, 3H), 0.93 (s, 3H); ${ }^{13} \mathrm{C} \mathrm{NMR}$ $\left(100 \mathrm{MHz}, \mathrm{CDCl}_{3}\right) \delta: 203.26,164.46(\mathrm{~d}, J=247.6 \mathrm{~Hz})$, 161.14, 134.45 (d, $J=8.0 \mathrm{~Hz}), 132.21(\mathrm{~d}, J=2.2 \mathrm{~Hz})$, $115.81,115.52$ (d, $J=21.3 \mathrm{~Hz}), 55.84,40.89,39.38,30.81$, 27.45, 26.19, 21.61.

(E)-3-(4-氯芐亚基)-6,6-二甲基二环[3.1.1]庚烷-2-酮 (2e): 无色透明晶体, 产率 $85.5 \%$. m.p. $109.7 \sim 110.7{ }^{\circ} \mathrm{C}$ (lit. $\left.{ }^{[29]} 107 \sim 108{ }^{\circ} \mathrm{C}\right) ;{ }^{1} \mathrm{H}$ NMR $\left(400 \mathrm{MHz}, \mathrm{CDCl}_{3}\right) \delta: 7.64$ $(\mathrm{t}, J=4.5 \mathrm{~Hz}, 1 \mathrm{H}), 7.52 \sim 7.50(\mathrm{~m}, 2 \mathrm{H}), 7.38 \sim 7.36(\mathrm{~m}$, $2 \mathrm{H}), 2.94(\mathrm{t}, J=2.8 \mathrm{~Hz}, 2 \mathrm{H}), 2.71(\mathrm{t}, J=5.6 \mathrm{~Hz}, 1 \mathrm{H})$, $2.65 \sim 2.62(\mathrm{~m}, 1 \mathrm{H}), 2.36 \sim 2.37(\mathrm{~m}, 1 \mathrm{H}), 1.49(\mathrm{~d}, J=10.5$ $\mathrm{Hz}, 1 \mathrm{H}), 1.38$ (s, 3H), 0.92 (s, 3H); ${ }^{13} \mathrm{C}$ NMR (100 MHz, $\mathrm{CDCl}_{3}$ ) $\delta: 202.91,134.74,134.18,134.10,133.10,131.78$, $128.73,55.78,40.82,39.29,30.78,27.36,26.12,21.55$.

(E)-6,6-二甲基-3-(4-硝基芐亚基)双环[3.1.1]庚烷-2酮 (2f): 黄色透明晶体, 产率 $62.7 \%$. m.p. 151.2 $151.6{ }^{\circ} \mathrm{C}\left(\right.$ lit. $\left.{ }^{[29]} 151 \sim 152{ }^{\circ} \mathrm{C}\right) ;{ }^{1} \mathrm{H}$ NMR $(400 \mathrm{MHz}$, $\left.\mathrm{CDCl}_{3}\right) \delta: 8.27(\mathrm{~s}, 1 \mathrm{H}), 8.24(\mathrm{~s}, 1 \mathrm{H}), 7.71(\mathrm{t}, J=4.7 \mathrm{~Hz}$, $3 \mathrm{H}), 2.98(\mathrm{~d}, J=2.5 \mathrm{~Hz}, 2 \mathrm{H}), 2.77 \sim 2.63(\mathrm{~m}, 2 \mathrm{H}), 2.41 \sim$ $2.37(\mathrm{~m}, 1 \mathrm{H}), 1.52(\mathrm{~d}, J=10.6 \mathrm{~Hz}, 1 \mathrm{H}), 1.40(\mathrm{~s}, 3 \mathrm{H}), 0.93$ (s, 3H); ${ }^{13} \mathrm{C}$ NMR (100 MHz, $\left.\mathrm{CDCl}_{3}\right) \delta: 202.51,147.26$, $142.02,136.41,132.91,131.01,123.70,55.85,41.06$, $39.20,30.87,27.34,26.16,21.68$.

\section{3 化合物 3 的制备}

取化合物 $2(1.5 \mathrm{mmol})$ 与氨基硫脲 $(1.5 \mathrm{mmol})$ 加入 到 $20 \mathrm{~mL}$ 无水乙醇中, 并在混合体系加入 $0.5 \mathrm{~mL}$ 的 $\mathrm{HCl}$ 作为催化剂加热回流. 反应完成后, 通过二氧化硅柱纯 化获得浓缩的反应溶液, 使用正己烷/乙酸乙酯 $(V: V=$ $8: 1)$ 洗脱, 得到化合物 $\mathbf{3 a} \sim \mathbf{3 f}$.

2-(3-芐亚基-6,6-二甲基双环[3.1.1]庚-2-亚基)肼-1硫代甲酰胺(3a): 淡黄色固体, 产率 80.1\%. m.p. 194.2 $197{ }^{\circ} \mathrm{C}$; ${ }^{1} \mathrm{H}$ NMR $\left(400 \mathrm{MHz}, \mathrm{CDCl}_{3}\right) \delta: 8.88$ (brs, $1 \mathrm{H}$ ), 7.49 (d, $J=7.6 \mathrm{~Hz}, 3 \mathrm{H}), 7.40$ (t, $J=7.7 \mathrm{~Hz}, 3 \mathrm{H}), 7.31$ (t, $J=7.4 \mathrm{~Hz}, 1 \mathrm{H}), 6.57(\mathrm{~s}, 1 \mathrm{H}), 3.14(\mathrm{t}, J=5.5 \mathrm{~Hz}, 1 \mathrm{H})$, $2.90 \sim 3.00(\mathrm{~m}, 2 \mathrm{H}), 2.54 \sim 2.60(\mathrm{~m}, 1 \mathrm{H}), 2.24(\mathrm{~s}, 1 \mathrm{H})$, 1.43 (s, 3H), 1.35 (d, $J=10.4 \mathrm{~Hz}, 1 \mathrm{H}), 0.91$ (s, 3H); ${ }^{13} \mathrm{C}$ NMR $\left(100 \mathrm{MHz}, \mathrm{CDCl}_{3}\right) \delta: 178.07,157.49,136.54$, $131.25,130.00,129.30,128.50,127.89,41.82,41.79$, 39.46, 31.81, 27.89, 26.18, 21.59; HRMS $\left(\mathrm{ESI}^{+}\right.$) calcd for $\mathrm{C}_{17} \mathrm{H}_{22} \mathrm{~N}_{3} \mathrm{~S}[\mathrm{M}+\mathrm{H}]^{+}$300.1521, found 300.1534 .

2-(6,6-二甲基-3-(4-甲基苄亚基)双环 [3.1.1]庚烷-2亚基)肼-1-硫代甲酰胺(3b): 淡黄色固体, 产率 67.2\%. m.p. $183 \sim 185{ }^{\circ} \mathrm{C} ;{ }^{1} \mathrm{H}$ NMR $\left(400 \mathrm{MHz}, \mathrm{CDCl}_{3}\right) \delta: 8.83$ (brs, 1H), 7.45 (s, 1H), 7.39 (d, $J=8.0 \mathrm{~Hz}, 3 \mathrm{H}), 7.21$ (d, $J=8.1 \mathrm{~Hz}, 2 \mathrm{H}), 6.49(\mathrm{~s}, 1 \mathrm{H}), 3.13(\mathrm{t}, J=4.7 \mathrm{~Hz}, 1 \mathrm{H})$, $2.90 \sim 3.00(\mathrm{~m}, 2 \mathrm{H}), 2.53 \sim 2.59(\mathrm{~m}, 1 \mathrm{H}), 2.39(\mathrm{~s}, 3 \mathrm{H})$, $2.24(\mathrm{~s}, 1 \mathrm{H}), 1.43(\mathrm{~s}, 3 \mathrm{H}), 1.34(\mathrm{~d}, J=10.5 \mathrm{~Hz}, 1 \mathrm{H}), 0.91$ (s, 3H); ${ }^{13} \mathrm{C}$ NMR $\left(100 \mathrm{MHz}, \mathrm{CDCl}_{3}\right) \delta: 178.48,157.61$, $137.98,133.76,130.96,130.02,129.44,129.26,41.84$, 41.74, 39.55, 31.92, 27.97, 26.21, 21.59, 21.40; HRMS $\left(\mathrm{ESI}^{+}\right.$) calcd for $\mathrm{C}_{18} \mathrm{H}_{24} \mathrm{~N}_{3} \mathrm{~S}[\mathrm{M}+\mathrm{H}]^{+} 314.1695$, found 314.1691.

2-(3-(4-甲氧基苠亚基)-6,6-二甲基双环 [3.1.1]庚-2亚基)肼-1-硫代甲酰胺(3c)：淡黄色固体，产率 79.5\%. m.p. 193 97 ${ }^{\circ} \mathrm{C} ;{ }^{1} \mathrm{H}$ NMR $\left(600 \mathrm{MHz}, \mathrm{CDCl}_{3}\right) \delta: 8.83$ (brs, 1H), 7.43 (d, $J=8.7 \mathrm{~Hz}, 2 \mathrm{H}), 7.42(\mathrm{~s}, 1 \mathrm{H}), 7.35$ (s, $1 \mathrm{H}), 6.92(\mathrm{~d}, J=8.7 \mathrm{~Hz}, 2 \mathrm{H}), 6.51(\mathrm{~s}, 1 \mathrm{H}), 3.84(\mathrm{~s}, 3 \mathrm{H})$, $3.11(\mathrm{t}, J=5.4 \mathrm{~Hz}, 1 \mathrm{H}), 2.88 \sim 2.94(\mathrm{~m}, 2 \mathrm{H}), 2.52 \sim 2.56$ (m, 1H), $2.23(\mathrm{~s}, 1 \mathrm{H}), 1.41(\mathrm{~s}, 3 \mathrm{H}), 1.33(\mathrm{~d}, J=10.3 \mathrm{~Hz}$, $1 \mathrm{H}), 0.89$ (s, 3H); ${ }^{13} \mathrm{C} \mathrm{NMR}\left(100 \mathrm{MHz}, \mathrm{CDCl}_{3}\right) \delta: 178.40$, $159.32,157.80,131.58,129.35,128.94,128.90,114.00$, 55.42, 41.75, 39.54, 31.94, 29.79, 27.97, 26.22, 21.59; HRMS ( $\mathrm{ESI}^{+}$) calcd for $\mathrm{C}_{18} \mathrm{H}_{24} \mathrm{~N}_{3} \mathrm{OS}[\mathrm{M}+\mathrm{H}]^{+} 330.1651$, found 330.1640 .

2-(3-(4-氟苄亚基)-6,6-二甲基双环[3.1.1]庚-2-亚基) 肼-1-硫代甲酰胺(3d): 淡黄色固体, 产率 82.7\%. m.p. $180 \sim 183{ }^{\circ} \mathrm{C} ;{ }^{1} \mathrm{H}$ NMR (400 MHz, $\mathrm{CDCl}_{3}$ ) $\delta: 8.83$ (brs, $1 \mathrm{H}), 7.46(\mathrm{dd}, J=5.5,8.7 \mathrm{~Hz}, 2 \mathrm{H}), 7.43(\mathrm{~s}, 1 \mathrm{H}), 7.37(\mathrm{~s}$, 1H), 7.09 (t, $J=8.7 \mathrm{~Hz}, 2 \mathrm{H}), 6.51(\mathrm{~s}, 1 \mathrm{H}), 3.13$ (t, $J=5.5$ $\mathrm{Hz}, 1 \mathrm{H}), 2.86 \sim 2.96(\mathrm{~m}, 2 \mathrm{H}), 2.54 \sim 2.60(\mathrm{~m}, 1 \mathrm{H}), 2.22 \sim$ $2.26(\mathrm{~m}, 1 \mathrm{H}), 1.43(\mathrm{~s}, 3 \mathrm{H}), 1.34(\mathrm{~d}, J=10.3 \mathrm{~Hz}, 1 \mathrm{H}), 0.91$ (s, 3H); ${ }^{13} \mathrm{C}$ NMR $\left(100 \mathrm{MHz}, \mathrm{CDCl}_{3}\right) \delta: 178.59,162.19(\mathrm{~d}$, $J=247.6 \mathrm{~Hz}), 157.31,132.74$ (d, $J=3.4 \mathrm{~Hz}), 131.73$ (d, $J=8.0 \mathrm{~Hz}), 130.92(\mathrm{~d}, J=2.2 \mathrm{~Hz}), 128.13,115.56(\mathrm{~d}, J=$ $21.3 \mathrm{~Hz}), 41.83,41.81,39.47,31.75,27.91,26.20,21.60$; HRMS (ESI ${ }^{+}$) calcd for $\mathrm{C}_{17} \mathrm{H}_{21} \mathrm{FN}_{3} \mathrm{~S}[\mathrm{M}+\mathrm{H}]^{+}$318.1431, found 318.1440 .

2-(3-(4-氯芐亚基)-6,6-二甲基双环[3.1.1]庚-2-亚基) 肼-1-硫代酰胺 (3e): 淡黄色固体, 产率 78.7\%. m.p. $184 \sim 186{ }^{\circ} \mathrm{C} ;{ }^{1} \mathrm{H}$ NMR $\left(400 \mathrm{MHz}, \mathrm{CDCl}_{3}\right) \delta$ : 8.74 (brs, $1 \mathrm{H}), 7.42$ (d, $J=8.6 \mathrm{~Hz}, 2 \mathrm{H}), 7.41$ (s, 1H), 7.37 (d, $J=8.8$ $\mathrm{Hz}, 2 \mathrm{H}), 7.35$ (s, 1H), 6.31 (s, 1H), 3.11 (t, $J=5.5 \mathrm{~Hz}, 1 \mathrm{H})$, $2.86 \sim 2.94(\mathrm{~m}, 2 \mathrm{H}), 2.55 \sim 2.65(\mathrm{~m}, 1 \mathrm{H}), 2.24 \sim 2.28(\mathrm{~m}$, 1H), 1.44 (s, 3H), 1.35 (d, $J=10.3 \mathrm{~Hz}, 1 \mathrm{H}), 0.91$ (s, 3H); ${ }^{13} \mathrm{C}$ NMR $\left(100 \mathrm{MHz}, \mathrm{CDCl}_{3}\right) \delta: 178.55,134.87,133.63$, 131.77, 131.08, 128.65, 127.94, 41.77, 41.61, 39.34, 31.69, $27.78,26.08,22.65,21.49$; HRMS $\left(\mathrm{ESI}^{+}\right)$calcd for 
$\mathrm{C}_{17} \mathrm{H}_{21} \mathrm{ClN}_{3} \mathrm{~S}[\mathrm{M}+\mathrm{H}]^{+}$334.1135, found 334.1145.

2-(6,6-二甲基-3-(4-硝基苄亚基)双环[3.1.1]庚-2-亚 基)肼-1-硫代甲酰胺(3f)：淡黄色固体，产率 81.3\%. m.p. $218 \sim 220{ }^{\circ} \mathrm{C}$; ${ }^{1} \mathrm{H}$ NMR (400 MHz, $\left.\mathrm{CDCl}_{3}\right) \delta$ : 8.81 (brs, $1 \mathrm{H}), 8.26(\mathrm{~d}, J=8.8 \mathrm{~Hz}, 2 \mathrm{H}), 7.63(\mathrm{~d}, J=8.8 \mathrm{~Hz}, 2 \mathrm{H}), 7.51$ $(\mathrm{s}, 1 \mathrm{H}), 7.36(\mathrm{~s}, 1 \mathrm{H}), 6.46(\mathrm{~s}, 1 \mathrm{H}), 3.15(\mathrm{t}, J=5.5 \mathrm{~Hz}, 1 \mathrm{H})$, $2.91 \sim 3.03(\mathrm{~m}, 2 \mathrm{H}), 2.59 \sim 2.65(\mathrm{~m}, 1 \mathrm{H}), 2.26 \sim 2.31(\mathrm{~m}$, $1 \mathrm{H}), 1.45$ (s, 3H), 1.36 (d, $J=10.4 \mathrm{~Hz}, 1 \mathrm{H}), 0.93$ (s, 3H); ${ }^{13} \mathrm{C}$ NMR $\left(100 \mathrm{MHz}, \mathrm{CDCl}_{3}\right) \delta: 178.95,156.17,146.69$, 143.01, 135.69, 130.46, 126.87, 123.83, 41.91, 41.80, 39.35, 32.04, 27.98, 26.14, 21.65; HRMS (ESI ${ }^{+}$) calcd for $\mathrm{C}_{17} \mathrm{H}_{21} \mathrm{~N}_{4} \mathrm{O}_{2} \mathrm{~S}[\mathrm{M}+\mathrm{H}]^{+}$345.1400, found 345.1385.

\section{4 化合物 SZ1 SZ24 的制备}

将等物质的量的含有多种取代基的溴代苯乙酮和 化合物 3 (1 mmol) 的混合物溶于 $20 \mathrm{~mL}$ 乙醇中并于室温 下搅拌 $2 \mathrm{~h}$. 在反应过程中, 反应液逐渐浑浊并形成悬 浮液. 过滤粉末, 用 $5 \mathrm{~mL}$ 石油醚洗涤, 得到目标化合物

\section{SZ1 $\sim$ SZ24.}

2-(2-(3-芐亚基-6,6-二甲基双环[3.1.1]庚烷-2-亚基) 肼基)-4-苯基噻唑(SZ1)：黄色固体，产率 77.6\%. m.p. $177.3 \sim 177.8{ }^{\circ} \mathrm{C} ;{ }^{1} \mathrm{H}$ NMR $\left(400 \mathrm{MHz}, \mathrm{CDCl}_{3}\right) \delta: 12.59$ (s, $1 \mathrm{H}), 7.70$ (d, $J=7.0 \mathrm{~Hz}, 2 \mathrm{H}), 7.50$ (d, $J=8.0 \mathrm{~Hz}, 2 \mathrm{H}), 7.44$ $(\mathrm{d}, J=8.0 \mathrm{~Hz}, 2 \mathrm{H}), 7.39(\mathrm{t}, J=8.6 \mathrm{~Hz}, 2 \mathrm{H}) 7.30(\mathrm{t}, J=$ $7.3 \mathrm{~Hz}, 1 \mathrm{H}), 6.74(\mathrm{~s}, 1 \mathrm{H}), 3.60(\mathrm{t}, J=5.3 \mathrm{~Hz}, 1 \mathrm{H}), 3.00 \sim$ 2.89 (m, 2H), $2.62(\mathrm{dt}, J=10.7,5.7 \mathrm{~Hz}, 1 \mathrm{H}), 2.37$ (s, 3H), $2.22(\mathrm{~s}, 1 \mathrm{H}), 1.50$ (s, 3H), 1.34 (d, $J=10.4 \mathrm{~Hz}, 1 \mathrm{H}), 0.94$ $(\mathrm{s}, 3 \mathrm{H}) ;{ }^{13} \mathrm{C}$ NMR $\left(100 \mathrm{MHz}, \mathrm{CDCl}_{3}\right) \delta: 169.70,166.17$, $140.91,136.68,131.31,131.01,130.71,130.46,129.96$, 128.84, 128.49, 125.97, 101.13, 44.83, 42.66, 39.73, 32.06, 28.19, 26.41, 21.98; HRMS (ESI ${ }^{+}$) calcd for $\mathrm{C}_{25} \mathrm{H}_{26} \mathrm{~N}_{3} \mathrm{~S}$ $[\mathrm{M}+\mathrm{H}]^{+}$400.1849, found 400.1853.

2-(2-(6,6-二甲基-3-(4-甲基苠亚基)双环[3.1.1]庚烷2-亚基)肼基)-4-苯基噻唑(SZ2)：黄色固体，产率 85.3\%. m.p. 163.6 164.5 ${ }^{\circ} \mathrm{C} ;{ }^{1} \mathrm{H}$ NMR (400 MHz, $\left.\mathrm{CDCl}_{3}\right) \delta$ : $12.47(\mathrm{~s}, 1 \mathrm{H}), 7.61(\mathrm{~d}, J=7.1 \mathrm{~Hz}, 2 \mathrm{H}), 7.42$ (s, 1H), 7.39 $7.28(\mathrm{~m}, 5 \mathrm{H}), 7.12(\mathrm{~d}, J=7.9 \mathrm{~Hz}, 2 \mathrm{H}), 6.66(\mathrm{~s}, 1 \mathrm{H}), 3.51(\mathrm{t}$, $J=5.4 \mathrm{~Hz}, 1 \mathrm{H}), 2.84(\mathrm{~s}, 2 \mathrm{H}), 2.53(\mathrm{dt}, J=10.3,5.6 \mathrm{~Hz}$, $1 \mathrm{H}), 2.28(\mathrm{~s}, 3 \mathrm{H}), 2.14(\mathrm{tt}, J=5.8,3.1 \mathrm{~Hz}, 1 \mathrm{H}), 1.41(\mathrm{~s}$, $3 \mathrm{H}), 1.25(\mathrm{~d}, J=10.4 \mathrm{~Hz}, 1 \mathrm{H}), 0.85(\mathrm{~s}, 3 \mathrm{H}) ;{ }^{13} \mathrm{C}$ NMR $(101$ $\left.\mathrm{MHz}, \mathrm{CDCl}_{3}\right) \delta: 169.09,165.76,140.31,138.08,133.36$, $130.74,130.13,129.96,129.49,129.40,129.05,127.25$, 125.43, 100.68, 44.26, 42.06, 39.22, 31.64, 27.71, 25.89, 21.44, 21.20, 18.26; HRMS (ESI ${ }^{+}$) calcd for $\mathrm{C}_{26} \mathrm{H}_{28} \mathrm{~N}_{3} \mathrm{~S}$ $[\mathrm{M}+\mathrm{H}]^{+}$414.2003, found 414.2004.

2-(2-(3-(4-甲氧基苄亚基)-6,6-二甲基双环[3.1.1]庚
烷-2-亚基)肼基)-4-苯基噻唑(SZ3)：黄色固体，产率 83.6\%. m.p. $180.8 \sim 181.9{ }^{\circ} \mathrm{C} ;{ }^{1} \mathrm{H}$ NMR $(400 \mathrm{MHz}$, $\left.\mathrm{CDCl}_{3}\right) \delta: 12.56(\mathrm{~s}, 1 \mathrm{H}), 7.71(\mathrm{~d}, J=6.8 \mathrm{~Hz}, 2 \mathrm{H}), 7.50(\mathrm{~d}$, $J=4.7 \mathrm{~Hz}, 2 \mathrm{H}), 7.48 \sim 7.39(\mathrm{~m}, 4 \mathrm{H}), 6.94(\mathrm{~d}, J=8.8 \mathrm{~Hz}$, 2H), $6.76(\mathrm{~s}, 1 \mathrm{H}), 3.85(\mathrm{~s}, 3 \mathrm{H}), 3.60(\mathrm{t}, J=5.4 \mathrm{~Hz}, 1 \mathrm{H})$, $2.93(\mathrm{~s}, 2 \mathrm{H}), 2.63(\mathrm{dt}, J=11.0,5.8 \mathrm{~Hz}, 1 \mathrm{H}), 2.24(\mathrm{~s}, 1 \mathrm{H})$, $1.51(\mathrm{~s}, 3 \mathrm{H}), 1.35$ (d, $J=10.3 \mathrm{~Hz}, 1 \mathrm{H}), 0.94(\mathrm{~s}, 3 \mathrm{H}) ;{ }^{13} \mathrm{C}$ NMR $\left(101 \mathrm{MHz}, \mathrm{CDCl}_{3}\right) \delta: 169.12,166.03,159.48$, $140.40,131.70,130.52,130.26,129.54,129.09,128.27$, 127.39 , 125.55, 113.96, 100.74, 55.35, 44.37, 42.17, 39.39, 31.81, 27.89, 26.05, 21.58; HRMS $\left(\mathrm{ESI}^{+}\right)$calcd for $\mathrm{C}_{26} \mathrm{H}_{28} \mathrm{~N}_{3} \mathrm{OS}[\mathrm{M}+\mathrm{H}]^{+}$430.1969, found 430.1974.

2-(2-(3-(4-氟苠亚基)-6,6-二甲基双环[3.1.1]庚烷-2亚基)肼基)-4-苯基噻唑(SZ4)：黄色固体，产率 74.2\%. m.p. $194.7 \sim 195.2{ }^{\circ} \mathrm{C} ;{ }^{1} \mathrm{H}$ NMR (400 MHz, $\left.\mathrm{CDCl}_{3}\right) \delta$ : $12.56(\mathrm{~s}, 1 \mathrm{H}), 7.68(\mathrm{~d}, J=6.9 \mathrm{~Hz}, 2 \mathrm{H}), 7.47 \sim 7.38(\mathrm{~m}$, $6 \mathrm{H}), 7.34$ (d, $J=8.5 \mathrm{~Hz}, 2 \mathrm{H}), 6.76(\mathrm{~s}, 1 \mathrm{H}), 3.59$ (t, $J=5.3$ $\mathrm{Hz}, 1 \mathrm{H}), 2.88$ (s, 2H), 2.60 (dd, $J=10.4,5.5 \mathrm{~Hz}, 1 \mathrm{H}), 2.21$ (s, 1H), $1.48(\mathrm{~s}, 3 \mathrm{H}), 1.30$ (d, $J=10.4 \mathrm{~Hz}, 1 \mathrm{H}), 0.91$ (s, $3 \mathrm{H}) ;{ }^{13} \mathrm{C} \mathrm{NMR}\left(101 \mathrm{MHz}, \mathrm{CDCl}_{3}\right) \delta: 169.49,165.49,162.4$ (d, $J=247.9 \mathrm{~Hz}), 140.70,134.48$ (d, $J=3.5 \mathrm{~Hz}), 131.43$ (d, $J=8 \mathrm{~Hz}), 130.49$ (d, $J=22.2 \mathrm{~Hz}), 129.73,129.68$, 128.83, 127.51, 125.73, 115.6 (d, $J=21.4 \mathrm{~Hz}), 101.12$, 44.59, 42.43, 39.44, 31.85, 27.99, 26.17, 21.75; HR-MS $\left(\mathrm{ESI}^{+}\right.$) calcd for $\mathrm{C}_{25} \mathrm{H}_{25} \mathrm{FN}_{3} \mathrm{~S}[\mathrm{M}+\mathrm{H}]^{+}$418.1751, found 418.1753.

2-(2-(3-(4-氯苄亚基)-6,6-二甲基双环 [3.1.1]庚烷-2亚基)肼基)-4-苯基噻唑(SZ5)：黄色固体，产率 77.6\%. m.p. $185.6 \sim 185.9{ }^{\circ} \mathrm{C} ;{ }^{1} \mathrm{H}$ NMR $\left(400 \mathrm{MHz}, \mathrm{CDCl}_{3}\right) \delta$ : $12.56(\mathrm{~s}, 1 \mathrm{H}), 7.62(\mathrm{~d}, J=6.9 \mathrm{~Hz}, 2 \mathrm{H}), 7.42 \sim 7.31(\mathrm{~m}$, $6 \mathrm{H}), 7.00(\mathrm{t}, J=8.7 \mathrm{~Hz}, 2 \mathrm{H}), 6.63(\mathrm{~s}, 1 \mathrm{H}), 3.52(\mathrm{t}, J=5.4$ $\mathrm{Hz}, 1 \mathrm{H}), 2.81$ (s, 2H), 2.55 (dt, $J=10.5,5.4 \mathrm{~Hz}, 1 \mathrm{H}), 2.14$ (s, 1H), $1.41(\mathrm{~s}, 3 \mathrm{H}), 1.25$ (d, $J=10.4 \mathrm{~Hz}, 1 \mathrm{H}), 0.85$ (s, $3 \mathrm{H}) .{ }^{13} \mathrm{C} \mathrm{NMR}\left(101 \mathrm{MHz}, \mathrm{CDCl}_{3}\right) \delta: 169.65,165.65$, $140.86,135.08,134.20,131.59$, 130.66, 129.89, 129.84, 128.99, 127.68, 125.89, 101.28, 44.75, 42.59, 39.61, 32.02, 28.15, 26.34, 21.92; HRMS (ESI ${ }^{+}$) calcd for $\mathrm{C}_{25} \mathrm{H}_{25} \mathrm{ClN}_{3} \mathrm{~S}$ $[\mathrm{M}+\mathrm{H}]^{+}$434.1521, found 434.1538.

2-(2-(6,6-二甲基-3-(4-硝基芐亚基)双环[3.1.1]庚烷2-亚基)肼基)-4-苯基噻唑(SZ6)：黄色固体，产率 87.5\%. m.p. 206.4 207.1 ${ }^{\circ} \mathrm{C} ;{ }^{1} \mathrm{H}$ NMR (400 MHz, $\left.\mathrm{CDCl}_{3}\right) \delta$ : $12.73(\mathrm{~s}, 1 \mathrm{H}), 8.23$ (d, $J=8.8 \mathrm{~Hz}, 2 \mathrm{H}), 7.70$ (d, $J=6.6 \mathrm{~Hz}$, 2H), $7.63(\mathrm{~d}, J=8.9 \mathrm{~Hz}, 2 \mathrm{H}), 7.55$ (s, 1H), 7.45 (dd, $J=$ $10.5,7.2 \mathrm{~Hz}, 3 \mathrm{H}), 6.78(\mathrm{~s}, 1 \mathrm{H}), 3.65(\mathrm{t}, J=5.4 \mathrm{~Hz}, 1 \mathrm{H})$, 3.01-2.88 (m, 2H), $2.66(\mathrm{dt}, J=10.4,5.3 \mathrm{~Hz}, 1 \mathrm{H}), 2.26$ (s, 
1H), 1.51 (s, 3H), 1.34 (d, $J=10.5 \mathrm{~Hz}, 1 \mathrm{H}), 0.94$ (s, 3H); ${ }^{13} \mathrm{C}$ NMR (101 MHz, $\left.\mathrm{CDCl}_{3}\right) \delta: 169.92,165.05,147.14$, $143.08,141.17,135.33,130.91,130.02,128.73,127.63$, 126.00, 124.08, 101.29, 44.90, 42.73, 39.57, 32.25, 28.24, 26.32, 22.00; HRMS (ESI ${ }^{+}$) calcd for $\mathrm{C}_{25} \mathrm{H}_{25} \mathrm{~N}_{4} \mathrm{O}_{2} \mathrm{~S}[\mathrm{M}+$ $\mathrm{H}]^{+}$445.1701, found 445.1696.

2-(2-(3-芐亚基-6,6-二甲基双环[3.1.1]庚烷-2-亚基) 肼基)-4-(4- 甲氧基苯基)噻唑(SZ7): 白色固体, 产率 83.3\%. m.p. $132.6 \sim 133.2{ }^{\circ} \mathrm{C} ;{ }^{1} \mathrm{H}$ NMR $(400 \mathrm{MHz}$, $\left.\mathrm{CDCl}_{3}\right) \delta: 12.52(\mathrm{~s}, 1 \mathrm{H}), 7.63(\mathrm{~d}, J=8.8 \mathrm{~Hz}, 2 \mathrm{H}), 7.51(\mathrm{~d}$, $J=7.2 \mathrm{~Hz}, 2 \mathrm{H}), 7.48$ (s, 1H), 7.39 (t, $J=7.6 \mathrm{~Hz}, 2 \mathrm{H}), 7.30$ (t, $J=7.3 \mathrm{~Hz}, 1 \mathrm{H}), 6.96(\mathrm{~d}, J=8.8 \mathrm{~Hz}, 2 \mathrm{H}), 6.57(\mathrm{~s}, 1 \mathrm{H})$, $3.81(\mathrm{~s}, 3 \mathrm{H}), 3.59(\mathrm{t}, J=5.3 \mathrm{~Hz}, 1 \mathrm{H}), 2.94(\mathrm{~s}, 2 \mathrm{H}), 2.62(\mathrm{dt}$, $J=9.7,5.2 \mathrm{~Hz}, 1 \mathrm{H}), 2.22(\mathrm{~s}, 1 \mathrm{H}), 1.49$ (s, 3H), 1.33 (d, $J=$ $10.4 \mathrm{~Hz}, 1 \mathrm{H}), 0.93$ (s, 3H); ${ }^{13} \mathrm{C} \mathrm{NMR}\left(101 \mathrm{MHz}, \mathrm{CDCl}_{3}\right) \delta$ : $169.59,165.86,161.49,140.79,136.73,131.19,131.06$, $130.46,128.84,128.46,127.52,120.45,115.33,98.96$, 55.84, 44.75, 42.63, 39.74, 32.07, 28.20, 26.42, 21.97; HRMS $\left(\mathrm{ESI}^{+}\right.$) calcd for $\mathrm{C}_{26} \mathrm{H}_{28} \mathrm{~N}_{3} \mathrm{OS}[\mathrm{M}+\mathrm{H}]^{+} 430.1954$, found 430.1957 .

2-(2-(6,6-二甲基-3-(4-甲基芐亚基)双环 [3.1.1]庚烷2-亚基)肼基)-4-(4-氟苯基)噻唑(SZ8): 白色固体, 产率 82.6\%. m.p. 207.6 208.4 ${ }^{\circ} \mathrm{C} ;{ }^{1} \mathrm{H}$ NMR $(400 \mathrm{MHz}$, $\left.\mathrm{CDCl}_{3}\right) \delta: 12.64(\mathrm{~s}, 1 \mathrm{H}), 7.77(\mathrm{~d}, J=8.8 \mathrm{~Hz}, 2 \mathrm{H}), 7.64$ (s, 1H), $7.54(\mathrm{~d}, J=8.0 \mathrm{~Hz}, 2 \mathrm{H}), 7.34(\mathrm{~d}, J=7.9 \mathrm{~Hz}, 2 \mathrm{H}), 7.12$ (s, 2H), $6.68(\mathrm{~s}, 1 \mathrm{H}), 3.96(\mathrm{~s}, 3 \mathrm{H}), 3.73(\mathrm{t}, J=5.4 \mathrm{~Hz}, 1 \mathrm{H})$, $3.07(\mathrm{~s}, 2 \mathrm{H}), 2.76(\mathrm{dt}, J=10.8,5.7 \mathrm{~Hz}, 1 \mathrm{H}), 2.51(\mathrm{~s}, 3 \mathrm{H})$, $2.35(\mathrm{~d}, \quad J=8.9 \mathrm{~Hz}, 1 \mathrm{H}), 1.63(\mathrm{~s}, 3 \mathrm{H}), 1.47$ (d, $J=10.4$ $\mathrm{Hz}, 1 \mathrm{H}), 1.07(\mathrm{~s}, 3 \mathrm{H}) ;{ }^{13} \mathrm{C}$ NMR $\left(101 \mathrm{MHz}, \mathrm{CDCl}_{3}\right) \delta$ : $168.94,165.65,158.65,140.61,140.47,138.19,133.53$, $130.83,130.12,129.62,129.19,127.05,119.11,116.68$, $58.41,42.22,39.37,31.78,27.84,26.01,21.59,21.35$, 18.29; HR-MS $\left(\right.$ ESI $^{+}$) calcd for $\mathrm{C}_{27} \mathrm{H}_{30} \mathrm{~N}_{3} \mathrm{OS}[\mathrm{M}+\mathrm{H}]^{+}$ 444.2113, found 444.2116.

2-(2-(3-(4-甲氧基苠亚基)-6,6-二甲基双环 [3.1.1]庚 烷-2-亚基)肼基)-4-(4-甲氧基苯基)噻唑(SZ9): 白色固 体, 产率 $82.1 \%$. m.p. 193.6 194.3 ${ }^{\circ} \mathrm{C}$; ${ }^{1} \mathrm{H}$ NMR (400 $\left.\mathrm{MHz}, \mathrm{CDCl}_{3}\right) \delta: 12.41(\mathrm{~s}, 1 \mathrm{H}), 7.55(\mathrm{~d}, J=8.9 \mathrm{~Hz}, 2 \mathrm{H})$, $7.44 \sim 7.35(\mathrm{~m}, 3 \mathrm{H}), 6.87(\mathrm{dd}, J=15.5,8.8 \mathrm{~Hz}, 4 \mathrm{H}), 6.47$ (s, 1H), 3.75 (d, $J=7.0 \mathrm{~Hz}, 6 \mathrm{H}), 3.50(\mathrm{t}, J=5.4 \mathrm{~Hz}, 1 \mathrm{H})$, $2.83(\mathrm{~s}, 2 \mathrm{H}), 2.54(\mathrm{dt}, J=10.9,5.8 \mathrm{~Hz}, 1 \mathrm{H}), 2.15(\mathrm{~s}, 1 \mathrm{H})$, $1.41(\mathrm{~s}, 3 \mathrm{H}), 1.26(\mathrm{~d}, J=10.3 \mathrm{~Hz}, 1 \mathrm{H}), 0.85(\mathrm{~s}, 3 \mathrm{H}) ;{ }^{13} \mathrm{C}$ NMR $\left(101 \mathrm{MHz}, \mathrm{CDCl}_{3}\right) \delta: 168.88,165.65,160.93$, $159.32,140.16,131.54,130.26,128.99,128.19,126.96$, $119.91,114.78,113.80,98.30,55.30,55.20,44.16,42.01$,
39.25, 31.68, 27.76, 25.91, 21.42; HRMS $\left(\mathrm{ESI}^{+}\right.$) calcd for $\mathrm{C}_{27} \mathrm{H}_{30} \mathrm{~N}_{3} \mathrm{O}_{2} \mathrm{~S}[\mathrm{M}+\mathrm{H}]^{+}$460.2055, found 460.2053.

2-(2-(3-(4-氟芐亚基)-6,6-二甲基二环[3.1.1]庚烷-2亚基)肼基)-4-(4-甲氧基苯基)噻唑(SZ10): 白色固体，产 率 82.3\%. m.p. 213.6 214.7 ${ }^{\circ} \mathrm{C}$; ${ }^{1} \mathrm{H}$ NMR $(400 \mathrm{MHz}$, $\left.\mathrm{CDCl}_{3}\right) \delta: 12.45(\mathrm{~s}, 1 \mathrm{H}), 7.53(\mathrm{~d}, J=8.8 \mathrm{~Hz}, 2 \mathrm{H}), 7.41 \sim$ $7.34(\mathrm{~m}, 3 \mathrm{H}), 6.98$ (t, $J=8.7 \mathrm{~Hz}, 2 \mathrm{H}), 6.87$ (d, $J=8.9 \mathrm{~Hz}$, $2 \mathrm{H}), 6.46(\mathrm{~s}, 1 \mathrm{H}), 3.72(\mathrm{~s}, 3 \mathrm{H}), 3.50(\mathrm{t}, J=5.3 \mathrm{~Hz}, 1 \mathrm{H})$, $2.80(\mathrm{~s}, 2 \mathrm{H}), 2.53(\mathrm{dt}, J=10.5,5.5 \mathrm{~Hz}, 1 \mathrm{H}), 2.13(\mathrm{~s}, 1 \mathrm{H})$, 1.40 (s, 3H), $1.23(\mathrm{~d}, J=10.4 \mathrm{~Hz}, 1 \mathrm{H}), 0.83(\mathrm{~s}, 3 \mathrm{H}) ;{ }^{13} \mathrm{C}$ NMR $\left(101 \mathrm{MHz}, \mathrm{CDCl}_{3}\right) \delta: 170.05,166.05,162.4(\mathrm{~d}, J=$ $248 \mathrm{~Hz}), 141.26,135.48$ (d, $J=3.4 \mathrm{~Hz}), 134.60$ (d, $J=8.1$ $\mathrm{Hz}), 131.53$ (d, $J=2.1 \mathrm{~Hz}), 130.29,130.24,129.39,128.08$ (d, $J=21.3 \mathrm{~Hz}), 126.29,101.68,45.15,42.99,40.01$, $32.42,28.55,26.74,22.32$. HR-MS $\left(\mathrm{ESI}^{+}\right)$calcd for $\mathrm{C}_{26} \mathrm{H}_{27} \mathrm{FN}_{3} \mathrm{OS}[\mathrm{M}+\mathrm{H}]^{+}$448.1865, found 448.1867.

2-(2-(3-(4-氯苄亚基)-6,6-二甲基双环 [3.1.1]庚烷-2亚基)肼基)-4-(4-甲氧基苯基)噻唑(SZ11): 白色固体, 产 率 83.5\%. m.p. 205.6 206.3 ${ }^{\circ} \mathrm{C}$; ${ }^{1} \mathrm{H}$ NMR (400 MHz, Pyridine- $\left.d_{5}\right) \delta: 8.19$ (d, $\left.J=8.7 \mathrm{~Hz}, 2 \mathrm{H}\right), 7.92(\mathrm{~s}, 1 \mathrm{H}), 7.65$ (d, $J=8.6 \mathrm{~Hz}, 2 \mathrm{H}), 7.59$ (d, $J=8.6 \mathrm{~Hz}, 2 \mathrm{H}), 7.28$ (s, $1 \mathrm{H})$, 7.15 (d, $J=8.8 \mathrm{~Hz}, 2 \mathrm{H}), 3.92$ (t, $J=5.6 \mathrm{~Hz}, 1 \mathrm{H}), 3.79$ (s, $3 \mathrm{H}), 2.99$ (d, $J=16.4 \mathrm{~Hz}, 1 \mathrm{H}), 2.90(\mathrm{~d}, J=16.9 \mathrm{~Hz}, 1 \mathrm{H})$, 2.59 (dt, $J=10.1,5.7 \mathrm{~Hz}, 1 \mathrm{H}), 2.17$ (s, 1H), 1.37 (s, 3H), $1.34(\mathrm{~s}, 1 \mathrm{H}), 0.97$ (s, 3H); ${ }^{13} \mathrm{C}$ NMR (101 MHz, Pyridine- $\left.d_{5}\right) \delta: 171.48,160.23,155.29,152.07,136.96,133.99$, $133.31,132.14,129.45,129.41,128.19,126.48,115.01$, 102.37, 55.81, 43.42, 41.73, 40.28, 32.71, 28.97, 26.46, 22.05; HRMS $\left(\mathrm{ESI}^{+}\right.$) calcd for $\mathrm{C}_{26} \mathrm{H}_{27} \mathrm{ClN}_{3} \mathrm{OS}[\mathrm{M}+\mathrm{H}]^{+}$ 464.1576, found 464.1581 .

2-(2-(6,6-二甲基-3-(4-硝基芐亚基)双环 [3.1.1]庚烷2-亚基)肼基)-4-(4-甲氧基苯基)噻唑(SZ12): 白色固体, 产率 83.8\%. m.p. $143.8 \sim 144.1{ }^{\circ} \mathrm{C}$; ${ }^{1} \mathrm{H}$ NMR $(400 \mathrm{MHz}$, $\left.\mathrm{CDCl}_{3}\right) \delta: 12.45(\mathrm{~s}, 1 \mathrm{H}), 7.90(\mathrm{~d}, J=6.8 \mathrm{~Hz}, 2 \mathrm{H}), 7.71(\mathrm{~s}$, $1 \mathrm{H}), 7.66$ (t, $J=7.2 \mathrm{~Hz}, 2 \mathrm{H}), 7.61$ (t, $J=7.4 \mathrm{~Hz}, 3 \mathrm{H}), 7.40$ (d, $J=8.0 \mathrm{~Hz}, 2 \mathrm{H}), 6.92(\mathrm{~s}, 1 \mathrm{H}), 3.78(\mathrm{t}, J=5.4 \mathrm{~Hz}, 1 \mathrm{H})$, 3.14 (s, 2H), 2.82 (dt, $J=10.4,5.6 \mathrm{~Hz}, 1 \mathrm{H}), 2.57$ (s, 3H), 2.42 (s, 1H), 1.69 (s, 3H), 1.54 (d, $J=10.4 \mathrm{~Hz}, 1 \mathrm{H}), 1.13$ (s, 3H); ${ }^{13} \mathrm{C}$ NMR $\left(101 \mathrm{MHz}, \mathrm{CDCl}_{3}\right) \delta: 169.41,166.18$, $166.09,159.09,159.01,140.90,138.63,133.97,131.27$, $130.56,130.06,129.63,119.55,117.12,58.85,42.66$, 39.81, 32.22, 28.27, 26.45, 22.02, 21.79, 18.81; HRMS $\left(\mathrm{ESI}^{+}\right)$calcd for $\mathrm{C}_{26} \mathrm{H}_{27} \mathrm{~N}_{4} \mathrm{O}_{3} \mathrm{~S}[\mathrm{M}+\mathrm{H}]^{+}$475.1706, found 475.1734 .

4-(2-(2-(3-芐亚基-6,6-二甲基双环[3.1.1]庚烷-2-亚 
基)肼基)噻唑-4-基)苯酚(SZ13)：黄色固体，产率 70.2\%. m.p. 132.6 133.2 ${ }^{\circ} \mathrm{C}$; ${ }^{1} \mathrm{H}$ NMR (400 MHz, Pyridine- $d_{5}$ ) $\delta$ : $9.02(\mathrm{~s}, 1 \mathrm{H}), 8.41(\mathrm{~d}, J=8.6 \mathrm{~Hz}, 2 \mathrm{H}), 8.25$ (s, 1H), 7.87 (d, $J=8.3 \mathrm{~Hz}, 3 \mathrm{H}), 7.42$ (s, 1H), 4.17 (q, $J=7.0 \mathrm{~Hz}, 1 \mathrm{H})$, $4.10(\mathrm{t}, J=5.5 \mathrm{~Hz}, 1 \mathrm{H}), 3.28 \sim 3.16(\mathrm{~m}, 2 \mathrm{H}), 2.80 \sim 2.75$ $(\mathrm{m}, 1 \mathrm{H}), 2.57(\mathrm{~s}, 3 \mathrm{H}), 2.37(\mathrm{~s}, 1 \mathrm{H}), 1.61(\mathrm{t}, J=7.0 \mathrm{~Hz}, 2 \mathrm{H})$, $1.56(\mathrm{~s}, 3 \mathrm{H}), 1.17(\mathrm{~s}, 3 \mathrm{H}) ;{ }^{13} \mathrm{C}$ NMR (101 MHz, Pyridine- $\left.d_{5}\right) \delta: 171.60,159.44,155.70,152.60,138.44,133.34$, $130.88,129.51,128.56,128.20,128.12,128.01,117.09$, 101.57, 43.53, 41.85, 40.44, 32.89, 29.05, 26.59, 22.16; HRMS $\left(\mathrm{ESI}^{+}\right.$) calcd for $\mathrm{C}_{25} \mathrm{H}_{26} \mathrm{~N}_{3} \mathrm{OS}[\mathrm{M}+\mathrm{H}]^{+} 416.1788$, found 416.1776 .

4-(2-(2-(6,6-二甲基-3-(4-甲基苄亚基)双环[3.1.1]庚 烷-2-亚基)肼基)噻唑-4-基)苯酚(SZ14): 黄色固体, 产率 73.4\%. m.p. 161.3 161.6 ${ }^{\circ} \mathrm{C}$; ${ }^{1} \mathrm{H}$ NMR (400 MHz, Pyridine- $\left.d_{5}\right) \delta: 8.22(\mathrm{~d}, J=8.6 \mathrm{~Hz}, 2 \mathrm{H}), 8.06(\mathrm{~s}, 1 \mathrm{H}), 7.69$ (d, $J=8.2 \mathrm{~Hz}, 3 \mathrm{H}), 7.40$ (t, $J=7.9 \mathrm{~Hz}, 4 \mathrm{H}), 7.23$ (s, 1H), 3.92 $(\mathrm{t}, J=5.6 \mathrm{~Hz}, 1 \mathrm{H}), 3.12 \sim 2.94(\mathrm{~m}, 2 \mathrm{H}), 2.59(\mathrm{dt}, J=10.2$, $5.8 \mathrm{~Hz}, 1 \mathrm{H}), 2.39$ (s, 3H), 2.18 (s, 1H), 1.39 (s, 1H), 1.37 $(\mathrm{s}, 3 \mathrm{H}), 0.98$ (s, 3H); ${ }^{13} \mathrm{C}$ NMR (101 MHz, Pyridine- $\left.d_{5}\right) \delta$ : $170.77,158.57,154.97,151.76,131.47,130.05,129.34$, $127.70,127.31,127.15,116.21,100.62,42.64,40.95$, 39.61, 32.12, 28.21, 25.74, 21.28, 20.95, 18.98; HRMS $\left(\mathrm{ESI}^{+}\right.$) calcd for $\mathrm{C}_{26} \mathrm{H}_{28} \mathrm{~N}_{3} \mathrm{OS}[\mathrm{M}+\mathrm{H}]^{+}$430.1941, found 430.1935 .

4-(2-(2-(3-(4-甲氧基苄亚基)-6,6-二甲基双环[3.1.1] 庚烷-2-亚基)肼基)噻唑-4-基)苯酚(SZ15)：黄色固体，产 率 72.7\%. m.p. 206.6 207.6 ${ }^{\circ} \mathrm{C}$; ${ }^{1} \mathrm{H}$ NMR (400 MHz, $\mathrm{CDCl} 3) \delta: 7.33 \sim 7.15(\mathrm{~m}, 4 \mathrm{H}), 7.11(\mathrm{~s}, 1 \mathrm{H}), 6.89(\mathrm{t}, J=$ $8.9 \mathrm{~Hz}, 2 \mathrm{H}), 6.70(\mathrm{~s}, 1 \mathrm{H}), 6.47$ (d, $J=8.7 \mathrm{~Hz}, 2 \mathrm{H}), 3.12(\mathrm{t}$, $J=5.4 \mathrm{~Hz}, 1 \mathrm{H}), 2.57(\mathrm{~d}, J=16.7 \mathrm{~Hz}, 1 \mathrm{H}), 2.46(\mathrm{~d}, J=16.6$ $\mathrm{Hz}, 1 \mathrm{H}), 2.23 \sim 2.06(\mathrm{~m}, 3 \mathrm{H}), 1.81(\mathrm{~s}, 1 \mathrm{H}), 1.02(\mathrm{~s}, 3 \mathrm{H})$, $0.85(\mathrm{~d}, J=10.0 \mathrm{~Hz}, 1 \mathrm{H}), 0.48(\mathrm{~s}, 3 \mathrm{H}) ;{ }^{13} \mathrm{C} \mathrm{NMR}(101$ $\mathrm{MHz}$, Pyridine- $\left.d_{5}\right) \delta: 171.56,159.89,159.30,155.98$, $152.55,132.29,131.12,130.87,128.48,128.15,127.67$, 116.95, 114.98, 101.34, 55.82, 43.41, 41.72, 40.44, 32.89, 29.01, 26.53, 22.05; HRMS (ESI ${ }^{+}$) calcd for $\mathrm{C}_{26} \mathrm{H}_{28} \mathrm{~N}_{3} \mathrm{O}_{2} \mathrm{~S}$ $[\mathrm{M}+\mathrm{H}]^{+}$446.1883, found 446.1871.

4-(2-(2-(3-(4-氟苠亚基)-6,6-二甲基双环[3.1.1]庚烷2-亚基)肼基)噻唑-4-基)苯酚(SZ16)：黄色固体, 产率 $81.5 \%$. m.p. $182.5 \sim 183.2{ }^{\circ} \mathrm{C} ;{ }^{1} \mathrm{H}$ NMR $(400 \mathrm{MHz}$, $\left.\mathrm{C}_{5} \mathrm{D}_{5} \mathrm{~N}\right) \delta: 8.24(\mathrm{~d}, J=8.6 \mathrm{~Hz}, 2 \mathrm{H}), 7.96(\mathrm{~s}, 1 \mathrm{H}), 7.71(\mathrm{dd}$, $J=5.6,8.6 \mathrm{~Hz}, 2 \mathrm{H}), 7.39$ (t, $J=8.6 \mathrm{~Hz}, 1 \mathrm{H}), 7.25(\mathrm{~s}, 1 \mathrm{H})$, $3.98(\mathrm{t}, J=5.6 \mathrm{~Hz}, 1 \mathrm{H}), 2.85(\mathrm{~s}, 1 \mathrm{H}), 2.87$ (s, 1H), 2.62 (s, $1 \mathrm{H}), 2.17$ (s, 1H), 1.49 (s, 3H), 1.40 (d, J=2.9, 1H), 0.99 $(\mathrm{s}, 3 \mathrm{H}) ;{ }^{13} \mathrm{C}$ NMR $\left(100 \mathrm{MHz}, \mathrm{C}_{5} \mathrm{D}_{5} \mathrm{~N}\right) \delta: 171.34,163.74(\mathrm{~d}$, $J=24.5 \mathrm{~Hz}), 161.21,159.13,155.31,152.32,134.70$ (d, $J=3.2 \mathrm{~Hz}), 132.61(\mathrm{~d}, J=2.2 \mathrm{~Hz}), 128.00$ (d, $J=7.8 \mathrm{~Hz})$, $126.63,117.02,115.83,115.68$ (d, $J=21.2 \mathrm{~Hz}), 101.43$, 41.69, 40.35, 32.53, 28.76, 26.36, 21.89; HRMS (ESI $\left.{ }^{+}\right)$ calcd for $\mathrm{C}_{25} \mathrm{H}_{25} \mathrm{FN}_{3} \mathrm{OS}[\mathrm{M}+\mathrm{H}]^{+}$434.1548, found 434.1540 .

4-(2-(2-(3-(4-氯苄亚基)-6,6-二甲基双环[3.1.1]庚烷2-亚基)肼基)噻唑-4-基)苯酚(SZ17)：黄色固体，产率 66.1\%. m.p. 206.6 207.3 ${ }^{\circ} \mathrm{C}$; ${ }^{1} \mathrm{H}$ NMR (400 MHz, Pyridine- $\left.d_{5}\right) \delta: 8.00(\mathrm{~d}, J=8.6 \mathrm{~Hz}, 2 \mathrm{H}), 7.83(\mathrm{~s}, 1 \mathrm{H}), 7.52(\mathrm{~d}$, $J=8.7 \mathrm{~Hz}, 2 \mathrm{H}), 7.17(\mathrm{~d}, J=8.6 \mathrm{~Hz}, 2 \mathrm{H}), 7.03 \sim 6.95(\mathrm{~m}$, $3 \mathrm{H}), 3.69(\mathrm{t}, J=5.6 \mathrm{~Hz}, 1 \mathrm{H}), 3.61(\mathrm{~s}, 3 \mathrm{H}), 2.90 \sim 2.72(\mathrm{~m}$, $2 \mathrm{H}), 2.42 \sim 2.32(\mathrm{~m}, 1 \mathrm{H}), 1.97(\mathrm{td}, J=5.8,3.0 \mathrm{~Hz}, 1 \mathrm{H})$, $1.18(\mathrm{~s}, 1 \mathrm{H}), 1.15(\mathrm{~s}, 3 \mathrm{H}), 0.76(\mathrm{~s}, 3 \mathrm{H}) ;{ }^{13} \mathrm{C}$ NMR $(101$ $\mathrm{MHz}$, Pyridine- $\left.d_{5}\right) \delta: 171.21,159.18,154.95,152.33$, $136.80,133.81,133.10,131.94,129.23,128.28,127.84$, 126.24, 116.78, 101.26, 43.19, 41.52, 40.09, 32.51, 28.77, 26.25, 21.83; HRMS (ESI ${ }^{+}$) calcd for $\mathrm{C}_{25} \mathrm{H}_{25} \mathrm{ClN}_{3} \mathrm{OS}[\mathrm{M}+$ $\mathrm{H}]^{+} 450.1393$, found 450.1370 .

4-(2-(2-(6,6-二甲基-3-(4-硝基苠亚基)双环[3.1.1]庚 烷-2-亚基)肼基)噻唑-4-基)苯酚(SZ18)：黄色固体，产率 64.2\%. m.p. 132.6 133.2 ${ }^{\circ} \mathrm{C}$; ${ }^{1} \mathrm{H}$ NMR (400 MHz, Pyridine- $\left.d_{5}\right) \delta: 8.33(\mathrm{~d}, J=8.8 \mathrm{~Hz}, 2 \mathrm{H}), 8.12(\mathrm{~d}, J=8.6 \mathrm{~Hz}$, 2H), 7.88 (s, 1H), 7.71 (d, $J=8.6 \mathrm{~Hz}, 2 \mathrm{H}), 7.32$ (d, $J=8.6$ $\mathrm{Hz}, 2 \mathrm{H}), 7.17$ (s, 1H), 3.85 (t, $J=5.6 \mathrm{~Hz}, 1 \mathrm{H}), 3.03 \sim 2.93$ (m, $1 \mathrm{H}), 2.88(\mathrm{dt}, J=17.1,3.0 \mathrm{~Hz}, 1 \mathrm{H}), 2.54(\mathrm{dtd}, J=10.2$, 6.0, $1.8 \mathrm{~Hz}, 1 \mathrm{H}), 2.12$ (tt, $J=6.0,3.0 \mathrm{~Hz}, 1 \mathrm{H}), 1.29$ (s, 3H), $1.27(\mathrm{~s}, 1 \mathrm{H}), 0.89(\mathrm{~s}, 3 \mathrm{H}) ;{ }^{13} \mathrm{C}$ NMR (101 MHz, Pyridine- $\left.d_{5}\right) \delta: 170.91,159.12,154.23,152.19,146.57,144.47$, $137.56,130.83,128.19,127.60,125.24,116.72,101.38$, 43.11, 41.41, 39.93, 32.71, 28.80, 26.10, 21.76; HRMS $\left(\mathrm{ESI}^{+}\right.$) calcd for $\mathrm{C}_{25} \mathrm{H}_{25} \mathrm{~N}_{4} \mathrm{O}_{3} \mathrm{~S}[\mathrm{M}+\mathrm{H}]^{+}$461.1642, found 461.1642 .

2-(2-(3-苠亚基-6,6-二甲基双环[3.1.1]庚-2-亚基)肼 基)-4-(4-氟苯基)噻唑(SZ19)：黄色固体，产率 83.9\%. m.p. 205.3 $206.1{ }^{\circ} \mathrm{C} ;{ }^{1} \mathrm{H}$ NMR (400 MHz, $\left.\mathrm{CDCl}_{3}\right) \delta$ : $12.54(\mathrm{~s}, 1 \mathrm{H}), 7.71(\mathrm{dd}, J=8.8,5.0 \mathrm{~Hz}, 2 \mathrm{H}), 7.52$ (d, $J=$ $8.7 \mathrm{~Hz}, 2 \mathrm{H}), 7.49$ (s, 1H), $7.40(\mathrm{t}, J=7.6 \mathrm{~Hz}, 2 \mathrm{H}), 7.31(\mathrm{t}$, $J=7.3 \mathrm{~Hz}, 1 \mathrm{H}), 7.17(\mathrm{t}, J=8.5 \mathrm{~Hz}, 2 \mathrm{H}), 6.68(\mathrm{~s}, 1 \mathrm{H}), 3.58$ (t, $J=5.3 \mathrm{~Hz}, 1 \mathrm{H}), 2.95(\mathrm{~s}, 2 \mathrm{H}), 2.63(\mathrm{dt}, J=10.7,5.7 \mathrm{~Hz}$, $1 \mathrm{H}), 2.23$ (s, 1H), 1.50 (s, 3H), 1.35 (d, $J=10.4 \mathrm{~Hz}, 1 \mathrm{H})$, $0.94(\mathrm{~s}, 3 \mathrm{H}) ;{ }^{13} \mathrm{C} \mathrm{NMR}\left(101 \mathrm{MHz}, \mathrm{CDCl}_{3}\right) \delta: 169.41$, 166.02, 163.79 (d, $J=3.4 \mathrm{~Hz}), 139.67,136.37,131.11$, 130.68, 130.17, 128.56, 128.23, 127.89 (d, $J=8.6 \mathrm{~Hz}$ ), 
123.86 (d, $J=3.4 \mathrm{~Hz}), 116.92$ (d, $J=22.2 \mathrm{~Hz}), 100.54$ (d, $J=1.7 \mathrm{~Hz}), 44.53,42.40,39.45,31.77,27.90,26.15$, 21.69; HRMS $\left(\mathrm{ESI}^{+}\right.$) calcd for $\mathrm{C}_{25} \mathrm{H}_{25} \mathrm{FN}_{3} \mathrm{~S}[\mathrm{M}+\mathrm{H}]^{+}$ 418.1760 , found 418.1761 .

2-(2-(6,6-二甲基-3-(4-甲基苄亚基)双环 [3.1.1]庚烷2-亚基)肼基)-4-(4-氟苯基)噻夾(SZ20): 黄色固体, 产率 $79.0 \%$. m.p. $177.0 \sim 177.8{ }^{\circ} \mathrm{C} ;{ }^{1} \mathrm{H}$ NMR $(400 \mathrm{MHz}$, $\left.\mathrm{CDCl}_{3}\right) \delta: 12.48(\mathrm{~s}, 1 \mathrm{H}), 7.61(\mathrm{~d}, J=6.8 \mathrm{~Hz}, 2 \mathrm{H}), 7.42(\mathrm{~s}$, $1 \mathrm{H}), 7.40 \sim 7.29(\mathrm{~m}, 5 \mathrm{H}), 7.12(\mathrm{~d}, J=7.9 \mathrm{~Hz}, 2 \mathrm{H}), 6.66(\mathrm{~s}$, $1 \mathrm{H}), 3.61(\mathrm{q}, J=7.0 \mathrm{~Hz}, 2 \mathrm{H}), 3.51(\mathrm{t}, J=5.4 \mathrm{~Hz}, 1 \mathrm{H}), 2.84$ (s, 2H), 2.53 (dt, $J=10.5,5.7 \mathrm{~Hz}, 1 \mathrm{H}), 2.28(\mathrm{~s}, 3 \mathrm{H}), 2.14$ (s, 1H), 1.41 (s, 3H), 1.25 (d, $J=10.4 \mathrm{~Hz}, 1 \mathrm{H}), 1.13$ (t, $J=$ $7.0 \mathrm{~Hz}, 3 \mathrm{H}), 0.85$ (s, 3H); ${ }^{13} \mathrm{C}$ NMR (101 MHz, $\left.\mathrm{CDCl}_{3}\right) \delta$ : 169.32, 165.84, 163.70 (d, $J=250.3 \mathrm{~Hz}), 139.95,138.33$, $133.58,130.96,130.18,129.70,129.28,127.81$ (d, $J=9.5$ $\mathrm{Hz}), 124.13$ (d, $J=3.5 \mathrm{~Hz}), 116.83(\mathrm{~d}, J=22.2 \mathrm{~Hz}), 100.66$ (d, $J=1.7 \mathrm{~Hz}), 44.37,42.30,39.45,31.85,27.93,26.13$, 21.66, 21.42; HRMS (ESI ${ }^{+}$) calcd for $\mathrm{C}_{26} \mathrm{H}_{27} \mathrm{FN}_{3} \mathrm{~S}$ [M+ $\mathrm{H}]^{+}$432.1912, found 432.1917 .

4-(4-氟苯基)-2-(2-(3-(4-甲氧基苠亚基)-6,6-二甲基 双环 [3.1.1]庚烷-2-亚基)肼基)噻唑(SZ21): 黄色固体, 产率 82.7\%. m.p. $180.8 \sim 181.9{ }^{\circ} \mathrm{C} ;{ }^{1} \mathrm{H}$ NMR $(400 \mathrm{MHz}$, $\left.\mathrm{CDCl}_{3}\right) \delta: 12.40(\mathrm{~s}, 1 \mathrm{H}), 7.63(\mathrm{dd}, J=8.8,5.0 \mathrm{~Hz}, 2 \mathrm{H})$, 7.40 (d, $J=5.1 \mathrm{~Hz}, 2 \mathrm{H}), 7.37$ (s, 1H), 7.08 (t, $J=8.5 \mathrm{~Hz}$, $2 \mathrm{H}), 6.85(\mathrm{~d}, J=8.7 \mathrm{~Hz}, 2 \mathrm{H}), 6.59(\mathrm{~s}, 1 \mathrm{H}), 3.76(\mathrm{~s}, 3 \mathrm{H})$, $3.48(\mathrm{t}, J=5.4 \mathrm{~Hz}, 1 \mathrm{H}), 2.84$ (s, 2H), 2.54 (dt, $J=11.0,5.8$ $\mathrm{Hz}, 1 \mathrm{H}), 2.16$ (s, 1H), 1.41 (s, 3H), 1.26 (d, $J=10.4 \mathrm{~Hz}$, $1 \mathrm{H}), 0.85(\mathrm{~s}, 3 \mathrm{H}) ;{ }^{13} \mathrm{C} \mathrm{NMR}\left(101 \mathrm{MHz}, \mathrm{CDCl}_{3}\right) \delta: 168.99$, $166.11,162.27$ (d, $J=251 \mathrm{~Hz}), 159.38,139.32,131.58$, 130.49, 128.93, 128.10, 127.67 (d, $J=8.5 \mathrm{~Hz}), 123.65$ (d, $J=3.5 \mathrm{~Hz}), 116.66$ (d, $J=22.2 \mathrm{~Hz}), 113.83,100.27$ (d, $J=$ $1 \mathrm{~Hz}), 55.22$, 44.23, 42.07, 39.26, 31.67, 27.76, 25.93, 21.44. HRMS $\left(\mathrm{ESI}^{+}\right)$calcd for $\mathrm{C}_{26} \mathrm{H}_{28} \mathrm{~N}_{3} \mathrm{OS}[\mathrm{M}+\mathrm{H}]^{+}$ 448.1857, found 448.1860 .

2-(2-(3-(4-氟芐亚基)-6,6-二甲基双环 [3.1.1]庚烷-2亚基)肼基)-4-(4-氟苯基)噻唑(SZ22)：黄色固体, 产率 71.2\%. m.p. $196.2 \sim 196.9{ }^{\circ} \mathrm{C} ;{ }^{1} \mathrm{H}$ NMR (400 MHz, $\left.\mathrm{CDCl}_{3}\right) \delta: 12.25(\mathrm{~s}, 1 \mathrm{H}), 7.87(\mathrm{dd}, J=8.8,5.0 \mathrm{~Hz}, 2 \mathrm{H})$, $7.64(\mathrm{t}, J=7.0 \mathrm{~Hz}, 3 \mathrm{H}), 7.31(\mathrm{t}, J=8.5 \mathrm{~Hz}, 2 \mathrm{H}), 7.24(\mathrm{t}$, $J=8.7 \mathrm{~Hz}, 2 \mathrm{H}), 6.89$ (s, $1 \mathrm{H}), 3.73(\mathrm{t}, J=5.3 \mathrm{~Hz}, 1 \mathrm{H}), 3.06$ (s, $2 \mathrm{H}), 2.78(\mathrm{dt}, J=10.6,5.7 \mathrm{~Hz}, 1 \mathrm{H}), 2.39(\mathrm{~s}, 1 \mathrm{H}), 1.65$ (s, 3H), 1.49 (d, $J=10.4 \mathrm{~Hz}, 1 \mathrm{H}), 1.09$ (s, 3H); ${ }^{13} \mathrm{C} \mathrm{NMR}$ $\left(100 \mathrm{MHz}, \mathrm{CDCl}_{3}\right) \delta: 169.74,166.26,164.69$ (d, $J=25.1$ Hz), 162.20 (d, $J=24.9 \mathrm{~Hz}), 140.05,132.90$ (d, $J=3.4$ $\mathrm{Hz}), 132.27$ (d, $J=8.05 \mathrm{~Hz}), 132.23,130.68(\mathrm{~d}, J=2.02$
$\mathrm{Hz}), 130.22,128.16(\mathrm{~d}, J=8.59 \mathrm{~Hz}), 124.18,124.15$, 117.28 (d, $J=22.2 \mathrm{~Hz}), 115.95$ (d, $J=21.0 \mathrm{~Hz}), 100.77$, $44.88,42.75,39.75,32.04,28.24,26.47,22.01$; HRMS $\left(\mathrm{ESI}^{+}\right.$) calcd for $\mathrm{C}_{25} \mathrm{H}_{24} \mathrm{~F}_{2} \mathrm{~N}_{3} \mathrm{~S}[\mathrm{M}+\mathrm{H}]^{+}$436.1661, found 436.1668 .

2-(2-(3-(4-氯芐亚基)-6,6-二甲基双环 [3.1.1]庚烷-2亚基)肼基)-4-(4-氟苯基)噻唑(SZ23)：黄色固体, 产率 75.3\%. m.p. $199.2 \sim 200.3{ }^{\circ} \mathrm{C} ;{ }^{1} \mathrm{H}$ NMR $(400 \mathrm{MHz}$, $\left.\mathrm{CDCl}_{3}\right) \delta: 12.40(\mathrm{~s}, 1 \mathrm{H}), 7.55(\mathrm{dd}, J=8.8,5.0 \mathrm{~Hz}, 2 \mathrm{H})$, $7.31(\mathrm{~d}, J=9.0 \mathrm{~Hz}, 3 \mathrm{H}), 7.01(\mathrm{t}, J=8.6 \mathrm{~Hz}, 2 \mathrm{H}), 6.92(\mathrm{t}$, $J=8.7 \mathrm{~Hz}, 2 \mathrm{H}), 6.50(\mathrm{~s}, 1 \mathrm{H}), 3.42(\mathrm{t}, J=5.3 \mathrm{~Hz}, 1 \mathrm{H}), 2.74$ (s, 2H), 2.47 (dt, $J=10.5,5.5 \mathrm{~Hz}, 1 \mathrm{H}), 2.08(\mathrm{~s}, 1 \mathrm{H}), 1.34$ (s, 3H), 1.18 (d, $J=10.4 \mathrm{~Hz}, 1 \mathrm{H}), 0.77$ (s, 3H); ${ }^{13} \mathrm{C}$ NMR $\left(101 \mathrm{MHz}, \mathrm{CDCl}_{3}\right) \delta: 169.30,165.82,164.25(J=25.1$ $\mathrm{Hz}), 162.46,161.05,139.61,132.45,131.87,131.79$, $130.24,129.78,127.72 \quad(J=9.0 \mathrm{~Hz}), 123.72,116.18(J=$ $2.2 \mathrm{~Hz}), 100.32(J=1.6 \mathrm{~Hz}), 44.44,42.31,39.31,31.60$, 27.80, 26.02, 21.57. HRMS $\left(\mathrm{ESI}^{+}\right)$calcd for $\mathrm{C}_{25} \mathrm{H}_{24} \mathrm{Cl}-$ $\mathrm{FN}_{3} \mathrm{~S}[\mathrm{M}+\mathrm{H}]^{+} 452.1758$, found 418.1753 .

2-(2-(6,6-二甲基-3-(4-硝基芐亚基)双环 [3.1.1]庚烷2-亚基)肼基)-4-(4-氟苯基)噻唑(SZ24)：黄色固体, 产率 73.2\%. m.p. $207.8 \sim 208.5{ }^{\circ} \mathrm{C} ;{ }^{1} \mathrm{H}$ NMR $(400 \mathrm{MHz}$, $\left.\mathrm{CDCl}_{3}\right) \delta: 12.60(\mathrm{~s}, 1 \mathrm{H}), 8.23(\mathrm{~d}, J=8.8 \mathrm{~Hz}, 2 \mathrm{H}), 7.70(\mathrm{dd}$, $J=8.8,5.0 \mathrm{~Hz}, 2 \mathrm{H}), 7.63(\mathrm{~d}, J=8.8 \mathrm{~Hz}, 2 \mathrm{H}), 7.55(\mathrm{~s}, 1 \mathrm{H})$, 7.15 (t, $J=8.5 \mathrm{~Hz}, 2 \mathrm{H}), 6.74(\mathrm{~s}, 1 \mathrm{H}), 3.61$ (t, $J=5.3 \mathrm{~Hz}$, $1 \mathrm{H}), 3.01 \sim 2.88(\mathrm{~m}, 2 \mathrm{H}), 2.65(\mathrm{dt}, J=10.4,5.2 \mathrm{~Hz}, 1 \mathrm{H})$, 2.26 (s, 1H), 1.50 (s, 3H), 1.33 (d, $J=10.5 \mathrm{~Hz}, 1 \mathrm{H}), 0.93$ (s, 3H); ${ }^{13} \mathrm{C}$ NMR $\left(101 \mathrm{MHz}, \mathrm{CDCl}_{3}\right) \delta: 169.87,165.10$, $163.6(\mathrm{~d}, J=250.8 \mathrm{~Hz}), 147.13,143.02,140.10,135.24$, 130.90, 128.78, 128.13 (d, $J=8.5 \mathrm{~Hz}), 124.06,117.23$ (d, $J=22.2 \mathrm{~Hz}), 101.2(\mathrm{~d}, J=1.6 \mathrm{~Hz}), 44.84,42.73,39.54$, $32.21,28.20,26.31,21.98$; HRMS $\left(\mathrm{ESI}^{+}\right)$calcd for $\mathrm{C}_{25} \mathrm{H}_{25} \mathrm{~N}_{4} \mathrm{O}_{2} \mathrm{FS}[\mathrm{M}+\mathrm{H}]^{+}$445.1697, found 445.1696.

\section{$3.5 \alpha$-淀粉酶抑制率测定}

根据文献[3]报道, 使用浊度测量法测定这 24 种化 合物对 $\alpha$-淀粉酶的抑制活性. 将玉米淀粉溶解在 20 $\mathrm{mg} / \mathrm{mL}$ 的缓冲液中, 然后在 $100{ }^{\circ} \mathrm{C}$ 沸水浴下将溶液凝 胶化 $2.5 \mathrm{~min}$ 制备得到玉米淀粉溶液. 在 96 孔酶标板中 加入 $20 \mu \mathrm{L} \alpha$-淀粉酶溶液 $(0.2 \mathrm{mg} / \mathrm{mL})$, 然后加入 $20 \mu \mathrm{L}$ 配置好的多浓度的抑制剂, 在 $37{ }^{\circ} \mathrm{C}$ 下温育 $5 \mathrm{~min}$. 将第 一步配置好的淀粉溶液冷却后, 吸取 $60 \mu \mathrm{L}$ 注入每个孔 中引发反应. 使用酶标仪在 $660 \mathrm{~nm}$ 处测每个孔的浊度 变化 $2 \mathrm{~h}$, 使用下面的等式计算抑制百分比.

$$
\text { 抑制率 }(\%)=\frac{\mathrm{AUC}_{\text {sample }}-\mathrm{AUC}_{\text {control }}}{\mathrm{AUC}_{\text {sample }}} \times 100 \%
$$


其中, $\mathrm{AUC}_{\text {sample }}$ 为抑制曲线下的面积, $\mathrm{AUC}_{\text {control }}$ 为空白 曲线下的面积, $\mathrm{IC}_{50}$ 为在特定测定条件下产生 $50 \%$ 酶活 性抑制的抑制剂浓度.

\section{6 抑制类型实验}

根据文献 ${ }_{[11]}$ 报道, 定量测定可溶性淀粉释放的寡糖 量来确定 $\alpha$-淀粉酶的抑制类型. 将可溶性淀粉溶解在 $20 \mathrm{mg} / \mathrm{mL}$ 的缓冲液中, 然后在 $100{ }^{\circ} \mathrm{C}$ 沸水浴中将溶液 凝胶化 $2.5 \mathrm{~min}$ 制备得到淀粉溶液. 在 96 孔酶标板中加 入 $20 \mu \mathrm{L} \alpha$-淀粉酶溶液 $(0.2 \mathrm{mg} / \mathrm{mL})$, 然后加入 $20 \mu \mathrm{L}$ 配 置好的多浓度的抑制剂[通过用磷酸缓冲液(PBS) 替代抑 制剂来制备阴性对照], 在 $37{ }^{\circ} \mathrm{C}$ 下预温育 $5 \mathrm{~min}$. 在每 个孔中加入 $60 \mu \mathrm{L}$ 刚才配置好的淀粉溶液来引发反应, 并在 $37{ }^{\circ} \mathrm{C}$ 下孵育 $5 \mathrm{~min}$. 然后通过加入 $100 \mu \mathrm{L}$ 的二硝 基水杨酸试剂(DNSA)终止反应，接着在沸水浴中加热 5 min 以显色. 然后将板整个置于冰水浴中冷却至室温后, 使用酶标仪在 $540 \mathrm{~nm}$ 处读取混合物的吸光度.

\section{7 分子对接}

通过 chemdrew 3D 程序绘制化合物, 并使用 MM2 方法优化它们的几何结构 ${ }^{[38]}$, 所有优化的配体均保存 为 $\mathrm{pdb}$ 形式, 用 AutodockTools 确定化合物分子的可旋 转键及扭转角度并加电荷处理, 并保存为 pdbqt 格式文 件以便与 $3 \mathrm{bc} 9$ 蛋白进行分子对接研究. 从 Protein Data Bank 数据库中下载 PDBID:3bc9 的蛋白晶体作为受体 结构, 在对接之前, 使用 Pymol 和 AutodockTools 软件去 除水分子及杂原子和加氢、加电荷、加力场等方式来优 化蛋白质结构. 除使用 AutodockTools 外, 用 Gride Box 模块定义活性腔: 以(19.119, 7.013, 9.282)为中心定义格 点盒子, 大小为 $18 \times 18 \times 18$, 格点间距为 $0.1 \mathrm{~nm}$. 使用 AutoDock Vina 分子对接工具将制备好的化合物分子对 接到 $\alpha$-淀粉酶的活性位点 ${ }^{[39]}$.

辅助材料(Supporting Information) 中间体化合物 3 的 ${ }^{1} \mathrm{H}$ NMR 和 ${ }^{13} \mathrm{C}$ NMR 谱图及产物 $\mathbf{S Z 1} \sim \mathbf{S Z 2 4}$ 的 ${ }^{1} \mathrm{H}$ NMR 和 ${ }^{13} \mathrm{C}$ NMR 谱图. 这些材料可以免费从本刊网站(http:// sioc-journal.cn/)上下载.

\section{References}

[1] Rathmann, W; Giani, G. Diabetes Care 2004, 27, 1047.

[2] Gupta, Y.; Tandon, N. Nat. Med. J. India 2016, 29, 189.

[3] Liu, T.; Song, L.; Wang, H. J. Agric. Food Chem. 2011, 59, 9756.

[4] Mellor, D. D.; Sathyapalan, T.; Kilpatrick, E. S. J. Agric. Food Chem. 2015, 63, 9910.
[5] Murotomi, K.; Umeno, A.; Yasunaga, M. J. Agric. Food Chem. 2015, 63, 6715 .

[6] Alberti, G.; Zimmet, P.; Shaw, J. Diabetes Care 2004, 27, 1798

[7] Fowler, M. J. Clin. Diabetes 2008, 26, 77.

[8] Jarald, E.; Joshi, S. B.; Jain, D. C. Iran. J. Pharmacol. Ther. 2008, $7,97$.

[9] Bhandari, M. R.; Jong-Anurakkun, N.; Hong, G. Food Chem. 2008, 106, 247.

[10] Camargo, A. C.; Regitano-d'Arce, M. A. B.; Biasoto, A. C. T. Food Chem. 2016, 212, 395.

[11] Zhang, Y.; Wong, A. I. C.; Wu, J. J. Funct. Foods 2016, 25, 568.

[12] Ma, Y. Y.; Zhao, D. G.; Zhou, A. Y. J. Agric. Food Chem. 2015, 63, 8162.

[13] Martinez-Gonzalez, A. I.; Díaz-Sánchez, Á. G. Spectrochim. Acta, Part A 2019, 206, 437.

[14] Taha, M.; Syukri, B. M.; Hadiani, I, N. Bioorg. Chem. 2018, 80, 36.

[15] Yousuf, S.; Khan, K. M.; Salar, U. Eur. J. Med. Chem. 2018, 159, 47.

[16] Figueiredo-González, M.; Reboredo-Rodríguez, P.; González-Barreiro, C. Food Res. Int. 2019, 116, 447.

[17] Sun, L.; Chen, W.; Meng, Y. Food Chem. 2016, 208, 51

[18] Hargrave, K. D.; Hess, F. K.; Oliver, J. T. J. Med. Chem. 1983, 26, 1158.

[19] Patt, W. C.; Hamilton, H. W.; Taylor, M. D. J. Med. Chem. 1992, $35,2562$.

[20] Kamble, R. D.; Meshram, R. J.; Hese, S. V. Comput. Biol. Chem. 2016, 61,86 .

[21] Jaen, J. C.; Wise, L. D.; Caprathe, B. W. J. Med. Chem. 1990, 33, 311.

[22] Mohammad, H.; Reddy, P. V. N.; Monteleone, D. Eur. J. Med. Chem. 2015, 94, 306

[23] Ergenç, N.; Çapan, G.; Günay, N. S. Arch. Pharm. 1999, 332, 343.

[24] Bell, F. W.; Cantrell, A. S.; Hoegberg, M. J. Med. Chem. 1995, 38, 4929.

[25] Badorc, A.; Bordes, M. F.; Cointet, P. J. Med. Chem. 1997, 40, 3393.

[26] Khan, K. M.; Qurban, S.; Salar, U. Bioorg. Chem. 2016, 68, 245.

[27] Wang, G.; Peng, Z.; Gong, Z. Bioorg. Chem. 2018, 78, 195.

[28] Paudel, Y. N.; Ali, M. R.; Shah, S. Biomed. Pharmacother. 2017, $89,651$.

[29] Rui, J.; Zhang, Q.; Wang, X. Chin. J. Org. Chem. 2017, 37, 218 (in Chinese). (芮坚, 张齐, 王欣, 有机化学, 2017, 37, 218.)

[30] Sun, N.; Wang, X.; Ding, Z. Chin. J. Org. Chem. 2016, 36, 2489 (in Chinese). (孙楠, 王欣, 丁志涁, 有机化学, 2016, 36, 2489.)

[31] Wang, Y.; Gu, W.; Shan, Y. Bioorg. Med. Chem. Lett. 2017, 27, 2360.

[32] Yang, J.; Xu, X.; Rui, J. Spectrochim. Acta, Part A 2017, 183, 60.

[33] Yang, J.; Xu, H.; Xu, X. Dyes Pigm. 2016, 128, 75.

[34] Wang, Y.; Wu, C.; Zhang, Q. Bioorg. Chem. 2019, 84, 468.

[35] Williams, L. K.; Zhang, X.; Caner, S. Nat. Chem. Biol. 2015, 92, 387.

[36] Akkarachiyasit, S.; Yibchokanun, S.; Wacharasindhu, S. Molecules 2011, 16, 2075.

[37] Liu B, Ao W W, Yang Y. J. Nanjing For. Univ., Nat. Sci. Ed. 2010, 34, 89 (in Chinese).

(刘兵, 敖汪伟, 杨杨，南京林业大学学报(自然科学版), 2010 , $34,89$.

[38] Bashary, R.; Khatik, G. L. Bioorg. Chem. 2018, 82, 156.

[39] O. Trott, A. J. Olson. J. Comput. Chem. 2010, 31, 455. 